\title{
Link-based system optimum dynamic traffic assignment problems with environmental objectives
}

by

\author{
Jiancheng Long \\ Professor \\ School of Automation and Transportation Engineering \\ Hefei University of Technology \\ Hefei 230601, China \\ Tel.: +86-551-63831101 \\ E-mail: jianchenglong@hfut.edu.cn

\section{Jiaxu Chen} \\ Master's student \\ School of Transportation Engineering \\ Hefei University of Technology \\ Hefei 230601, China \\ E-mail: chenjiaxu@mail.hfut.edu.cn

\section{W.Y. Szeto} \\ Assistant Professor \\ Department of Civil Engineering \\ The University of Hong Kong \\ Pokfulam Road, Hong Kong \\ Tel.: +852-28578552 \\ Email: ceszeto@hku.hk
}

\section{Qin Shi}

Professor

School of Transportation Engineering

Hefei University of Technology

Hefei 230601, China

Tel.: +86-551-62919162

E-mail: shiqin@hfut.edu.cn 


\begin{abstract}
Maintaining air-quality standards has been a priority for transportation planners and policy makers worldwide. However, most existing system optimum dynamic traffic assignment (SO-DTA) models do not accommodate environmental objectives. In this paper, we use the link transmission model (LTM) to develop SO-DTA models that minimize total system emissions (TSE) in single destination networks. We use step functions to approximate cumulative flow curves for individual links, and to decompose link inflow into sub-flows according to time intervals at which they leave the link. The decomposed link inflows are used to estimate link emissions. Dynamic network constraints, non-vehicle holding constraints and link decomposition constraints are considered, and SO-DTA problems with environmental objectives are formulated as mixed integer linear programming (MILP) problems. Any average speed based emission functions can be used for our models. Finally, numerical examples are provided to demonstrate the property of the proposed models.
\end{abstract}

Keywords: Dynamic traffic assignment; system optimum; link transmission model; emission. 


\section{Introduction}

Dynamic traffic assignment (DTA) has long been recognized as a key component of network planning and transport policy evaluation, in addition to real-time traffic operation and management (Szeto and Lo, 2006). System-optimum DTA (SO-DTA), a special case of DTA based on a dynamic extension of Wardrop's (1952) second principle, is used to predict a time-dependent traffic state with optimal network performance, and to provide a benchmark for controlling and managing dynamic traffic networks. For example, SO-DTA models are used in road congestion pricing (e.g., Yang and Meng, 1998; Carey and Watling, 2012), signal control (e.g., Lo, 2001; Lin and Wang, 2004), network design (e.g., Waller and Ziliaskopoulos, 2001; Waller et al., 2006), and emergency evacuation traffic management (e.g., Liu et al., 2006; Chiu et al., 2007).

Existing SO-DTA models can be classified into continuous-time models (e.g., Friesz et al., 1989; Chow, 2007, 2009a, b; Ma et al., 2014, 2015) and discrete-time models (e.g., Merchant and Nemhauser, 1978a, b; Carey, 1987; Ziliaskopoulos, 2000; Nie, 2011; Zheng and Chiu, 2011; Long and Szeto, 2015), depending on whether the modeling period is discretized into time steps. Both categories of model have advantages and disadvantages. Continuous-time models can provide analytical insights (such as the closed form externality analysis), but cannot be efficiently solved due to their complex structure (Nie, 2011). Discrete-time models are usually formulated as mathematical programming problems, such as linear programming (LP) problems (e.g., Ziliaskopoulos, 2000) and mixed integer linear programming (MILP) problems (e.g., Lin and Wang, 2004; Pavlis and Recker, 2009), and can thus be more easily solved than continuous-time SO-DTA models. However, this category of models also compromise computational tractability in large-scale network applications due to the presence of numerous decision variables and constraints.

There are three major types of objectives in existing SO-DTA models: minimizing total system travel time (TSTT) (e.g., Merchant and Nemhauser, 1978a, b; Carey, 1987; Ghali and Smith, 1995; Ziliaskopoulos, 2000; Li et al., 2003; Lin and Wang, 2004; Munoz and Laval, 2006; Shen et al., 2007; Chow, 2007, 2009a,b; Pavlis and Recker, 2009; Nie, 2011; Zheng and Chiu, 2011; Zhu and Ukkusuri, 2013; Ma et al., 2014; Long and Szeto, 2015; Zheng et al., 2015), minimizing total system emissions (TSE) (e.g., Aziz and Ukkusuri, 2012), and minimizing both TSTT and TSE for a whole network in an integrated manner (e.g., Aziz and Ukkusuri, 2012; Ma et al., 2015). Most existing SO-DTA models accommodate only network mobility, and are used to meet the first of the above objectives: to minimize the TSTT spent by travelers in a network. SO-DTA models designed to address TSTT are usually based on linear or convex functions of link flow. This leads to mathematical programming formulations that are computationally efficient and solvable for reasonably sized networks. The 
objective of minimizing TSE has long been integrated with static traffic assignment problems (e.g., Rilett and Benedek, 1994; Benedek and Rilett, 1998; Nagurney, 2000; Yin and Lawphongpanich, 2006). However, few researchers have considered SO-DTA problems in terms of TSE. To the best of our knowledge, Aziz and Ukkusuri (2012) were the first to propose a SO-DTA model with an environmental objective. Aziz and Ukkusuri (2012) integrated an emissions-based objective with a traditional SO-DTA framework, and formulated the SO-DTA problem in terms of TSE as a non-linear and non-convex mathematical function. The proposed model was further approximated by quadratic programming (QP) model. However, the accuracy of the approximation is only high when the network is very congested, and the model is limited to a particular carbon monoxide (CO) emission function. To address the third objective, SO-DTA models are developed with weighted TSTT and TSE components and capture the trade-off between emissions and travel time (e.g., Aziz and Ukkusuri, 2012; Ma et al., 2015).

Dynamic network constraints are generally used to formulate feasible domains for existing SO-DTA models. There are four categories of dynamic network constraints: mass balance constraints, flow conservation constraints, flow propagation constraints, and definitional constraints. The constraints used are highly dependent on the underlying DNL model, such as point queue models (Ban et al., 2012), exit flow models (e.g., Merchant and Nemhauser, 1978a, b; Carey and Srinivasan, 1993; Lam and Huang, 1995; Wie and Tobin, 2002), and advanced exit flow models (e.g., Kuwahara and Akamatsu, 2001; Lo and Szeto, 2002; Yperman, 2007; Nie, 2011; Meng and Khoo, 2012; Zheng et al., 2015). The traffic flow models used in DTA problems should also have certain desirable properties, such as queue spillback (e.g., Daganzo, 1995; Lo and Szeto, 2002; Szeto and Lo, 2004; Ma et al., 2014), first-in-first-out (FIFO) (e.g., Astarita, 1996; Huang and Lam, 2002; Long and Szeto, 2015), and non-vehicle holding (NVH) (e.g., Ziliaskopoulos, 2000; Shen et al., 2007; Nie, 2011; Zheng and Chiu, 2011; Zhu and Ukkusuri, 2013). Queue spillback occurs when the end of a queue spills backward in the network. This property can be easily captured in a SO-DTA model by incorporating a physical queue traffic flow model. FIFO implies that vehicles that enter a link earlier will leave it sooner (Wu et al. 1998; Lo and Szeto, 2002; Long et al., 2011). The FIFO constraint on traffic flow in a single-destination network is usually assumed to be satisfied by nature, and the constraint becomes necessary when multi-commodity flow is addressed. Vehicle holding $(\mathrm{VH})$ implies that drivers are reluctant to move forward from upstream links to downstream links even if there are vacant spaces in the downstream links. In many discrete SO-DTA models, the problem of VH stems from relaxation and linearization (e.g., Merchant and Nemhauser, 1978a; Carey and Subrahmanian, 2000; Ziliaskopoulos, 2000; Nie, 2011).

In this paper, we use step functions to approximate a cumulative-flow curve for each link, and 
decompose link inflow into sub-flows according to time of departure from the link. Based on the travel times estimated from the decomposed link inflows, we develop two methods of evaluating link emissions. In contrast with existing methods (e.g., Aziz and Ukkusuri, 2012; Ma et al., 2015), the proposed methods of estimating link emissions can be integrated with any average speed based emission functions. In addition, we use Yperman's (2007) link transmission model (LTM) to represent dynamic network constraints and NVH constraints on SO-DTA problems in single-destination networks. The LTM combines Daganzo's (1995) cell transmission model (CTM) with a triangular fundamental diagram and Newell's (1993) cumulative curves. As each link can be treated as a single cell, the LTM has a much higher computational efficiency than the Lighthill-Whitham-Richards model, a classical numerical solution scheme, while retaining the latter's accuracy (Yperman, 2007). We also develop link inflow decomposition constraints to represent the relationship between cumulative decomposed link flow and cumulative link inflow and outflow. Integrating the LTM-based dynamic network constraints, NVH constraints, link inflow decomposition constraints and TSE derived from the estimated link emissions, we formulate SO-DTA models with environmental objectives as MILP problems. Four SO-DTA models are proposed. Two of them are with single objective, and the other two models are with bi-objective.

The main contributions made in our research are as follows.

First, we develop a novel method of estimating link emissions and TSE using decomposed link inflow. The TSE obtained is a linear function with respect to cumulative link flow.

Second, we propose link inflow decomposition constraints for SO-DTA problems with environmental objectives, which represent the relationship between cumulative decomposed link inflow and cumulative link inflow and outflow.

Third, we develop link-based MILP formulations for SO-DTA problems with environmental objectives, in which link transmission model is used as the traffic flow model. The proposed link-based models are more accurate and computationally efficient than existing cell-based models, and can achieve a global optimum.

The rest of the paper is organized as follows. In the next section, we provide an overview of discrete-time link travel time and propose methods of estimating discrete-time link emissions. In Section 3, LTM-based dynamic network constraints, NVH constraints, and link inflow decomposition constraints are applied to SO-DTA models, and four SO-DTA problems with environmental objectives are formulated as MILP problems. Numerical examples are provided in Section 4, and conclusions are drawn in Section 5. 


\section{Estimation of link travel time and emissions}

\subsection{Continuous-time link travel times and emissions}

Let $U_{a}(t) \quad\left(V_{a}(t)\right)$ be the cumulative number of vehicles that enter (exit) link $a$ at time $t$. As shown in Fig. 1, link travel time is related to cumulative vehicle number as follows: $U_{a}(t)=V_{a}\left(t+\tau_{a}(t)\right)$, where $\tau_{a}(t)$ is the link travel time with respect to time $t$. If $U_{a}(t)$ and $V_{a}(t)$ are strictly increasing with respect to $t$ and the FIFO condition is met, the dynamic link travel time can be formulated as follows (Long et al., 2011):

$$
\tau_{a}(t)=V_{a}^{-1}\left(U_{a}(t)\right)-t,
$$

where $V_{a}^{-1}(\cdot)$ is the inverse function of $V_{a}(\cdot)$.

Following Penic and Upchurch (1992), the emission rate of a link is assumed to be a function of the average speed of the vehicles traveling through the link, i.e. $L_{a} / \tau_{a}(t)$. We thus obtain the total emissions caused by travelers using link $a$ for the whole period under study as follows:

$$
E_{a}=\int_{0}^{T} \phi_{a}\left(L_{a} / \tau_{a}(t)\right) \tau_{a}(t) u_{a}(t) d t
$$

where $\phi_{a}(\cdot)$ is the emission-rate function for link $a$, measured in grams per vehicle per second, $T$ is the period under study, and $u_{a}(t)$ is the rate of inflow to link $a$ at time $t$.

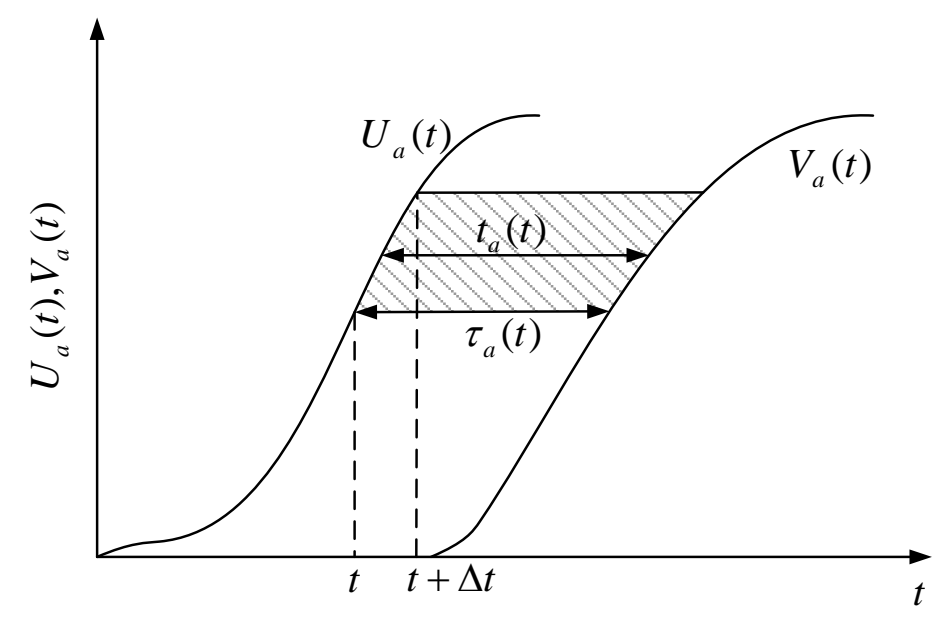

Fig. 1. Cumulative vehicle numbers as a function of time.

\subsection{Estimation of discrete-time link travel time}

In DTA models, time $t$ is usually discretized into small time intervals. In this paper, $\Delta t$ denotes interval length. The average travel time of the vehicles entering a link during interval $(t, t+\Delta t]$ can be calculated as follows (Long et al., 2011):

$$
t_{a}(t)=\frac{\int_{U_{a}(t)}^{U_{a}(t+\Delta t)}\left[V_{a}^{-1}(v)-U_{a}^{-1}(v)\right] d v}{U_{a}(t+\Delta t)-U_{a}(t)},
$$

where $U_{a}^{-1}(\cdot)$ is the inverse function of $U_{a}(\cdot)$. The numerator on the right hand side of Eq. (3) is equal to the shadowed area in Fig. 1, and the denominator is the number of vehicles that enter link $a$ 
during this interval.

We discretize a given period $T$ into a finite set of time intervals: $K=\{k=1,2, \cdots, \underline{K}\}$. Let $\delta$ be the interval length, such that $\delta \underline{K}=T$. To simplify the definition, let $U_{a}(k)$ and $V_{a}(k)$ be the cumulative numbers of vehicles that enter and leave link $a$ during interval $k$, respectively. As shown in Fig. 2, we use step functions to approximate the profiles of the cumulative flows $U_{a}(k)$ and $V_{a}(k)$ for link $a$. It is clear from the figure that not all of the vehicles that enter link $a$ during interval $k$ (i.e., $\left.y_{a}(k)\right)$ can exit link $a$ during the same discretized time tick; instead, the full packet must be decomposed to give several sub-packets that leave the link at different intervals. Let $y_{a}(k, l)$ be the number of vehicles entering link $a$ during interval $k$ and exiting the link during interval $l$, and let $Y_{a}(k, l)$ be the cumulative number of vehicles entering link $a$ during interval $k$ and exiting the link at the end of interval $l$. By definition, the following is true:

$$
\begin{aligned}
& y_{a}(k)=\int_{(k-1) \delta}^{k \delta} u_{a}(t) d t=U_{a}(k)-U_{a}(k-1), \forall k \in K, \\
& y_{a}(k)=\sum_{l \in K} y_{a}(k, l), \forall k \in K, \text { and } \\
& y_{a}(k, l)=Y_{a}(k, l)-Y_{a}(k, l-1), \forall k \in K, l \in K .
\end{aligned}
$$

According to Long et al. (2011), the cumulative flow $Y_{a}(k, l)$ can be formulated as follows:

$$
Y_{a}(k, l)= \begin{cases}0, & \text { if } l<n_{a}^{k-1}, \\ V_{a}(l)-U_{a}(k-1), & \text { if } n_{a}^{k-1} \leq l<n_{a}^{k}, \\ U_{a}(k)-U_{a}(k-1), & \text { otherwise, }\end{cases}
$$

where $n_{a}^{k}$ is the critical outflow interval with respect to interval $k$, and is defined as follows:

$$
n_{a}^{k}=\min \left\{l \mid U_{a}(k) \leq V_{a}(l), l>k+\vec{\imath}_{a} / \delta\right\},
$$

where $\vec{\imath}_{a}$ is the free flow travel time of vehicles on link $a$, and we assume that $\vec{l}_{a} / \delta$ is an integer.

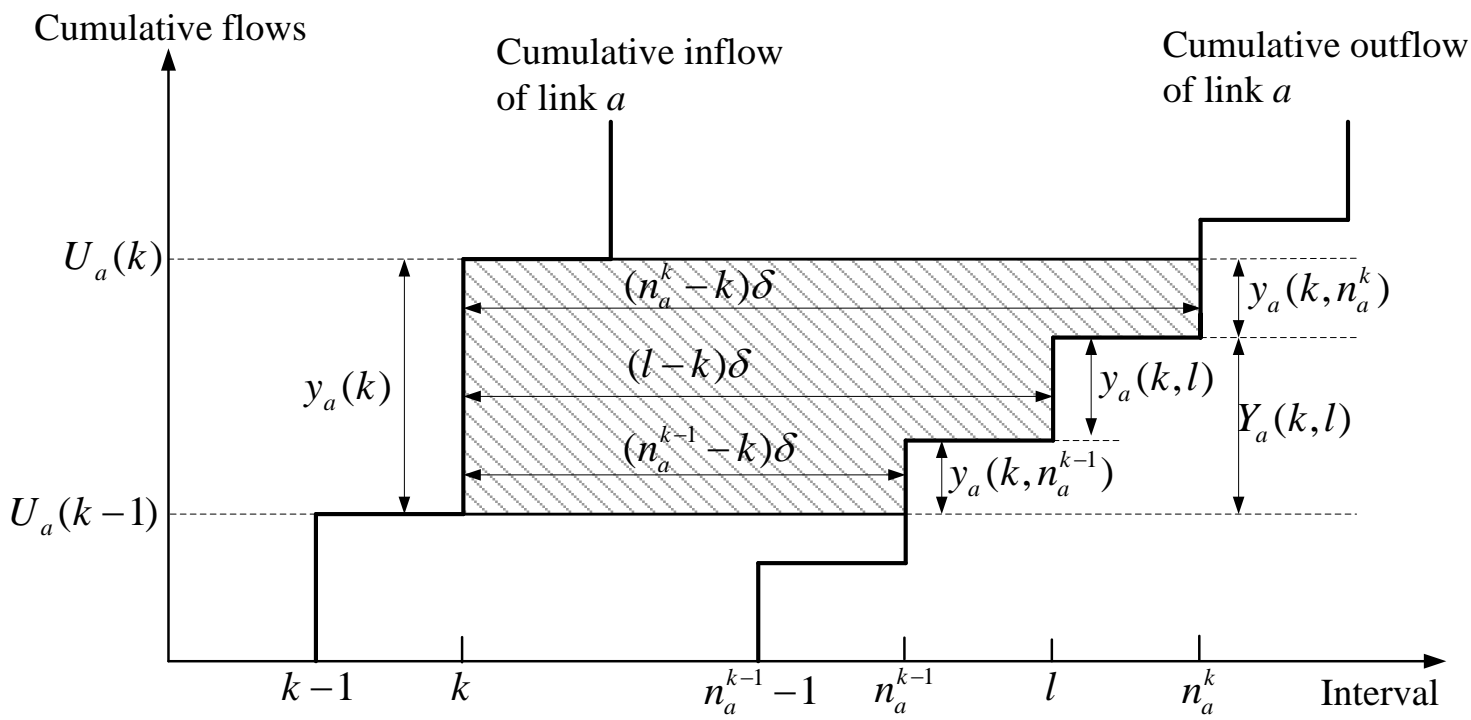


Fig. 2. Decomposition of link inflow.

As shown in Fig. 1, when the cumulative inflow has a steeper slope, and the cumulative outflow has a less steep one, the inflow in one time step can be divided into several time steps at the outflow side. In this case, we have $n_{a}^{k-1}<n_{a}^{k}$. We may also have another case: the cumulative inflow has a less steep slope and the outflow has a steeper slope. The inflow in one time step can leave the link during one time step at the outflow side. In this case, we have $n_{a}^{k-1}=n_{a}^{k}$.

Any sub-packet $y_{a}(k, l)$ that enters link $a$ during interval $k$ and leaves link $a$ during interval $l$ has an estimated travel time of $(l-k) \delta$ and a total travel time of $y_{a}(k, l)(l-k) \delta$. The total travel time of the entire packet $y_{a}(k)$, which is obtained by summing the travel times of individual sub-packets, is equal to the shadowed area in Fig. 2. Therefore, we can formulate the average travel time of the vehicles that enter link $a$ during interval $k$ as follows:

$$
t_{a}(k)=\sum_{l \in K} y_{a}(k, l)(l-k) \delta / y_{a}(k) .
$$

Substituting Eqs. (4) and (6) into Eq. (9) gives the following:

$$
t_{a}(k)=\sum_{l \in K} \frac{(l-k) \delta\left[Y_{a}(k, l)-Y_{a}(k, l-1)\right]}{\left[U_{a}(k)-U_{a}(k-1)\right]} .
$$

Assumption 1. The continuous-time link travel time is continuous with respect to time instant $t$.

Lemma 1. Under Assumption 1, the link travel times calculated by Eq. (10) converge to the continuous-time link travel times calculated by Eq. (1).

The proof is similar to the proof provided for Proposition 10 by Long et al. (2013). The detailed proof is presented in Appendix A.

Note that Assumption 1 may not be satisfied if queue spillback happens. In this case, the link travel times estimated by Eq. (10) will never converge to the continuous-time link travel times calculated by Eq. (1).

\subsection{Estimation of discrete-time link emissions}

\subsubsection{Estimate link emissions from entire packet}

We assume that all vehicles entering link $a$ during the same interval travel through the link at the same constant speed. The estimated travel time of the entire packet $y_{a}(k)$ can be used to estimate average vehicle speed, as follows:

$$
s_{a k}=\frac{L_{a}}{t_{a}(k)},
$$

where $L_{a}$ is the length of link $a$.

Therefore, the emission rate of the entire packet $y_{a}(k)$ can be obtained as follows:

$$
\phi_{a k}=\phi_{a}\left(s_{a k}\right)=\phi_{a}\left(L_{a} / t_{a}(k)\right) \text {. }
$$


We estimate the total emissions arising from travelers using link $a$ during the period under study by summing the total emissions for each packet, as follows:

$$
\bar{E}_{a}=\sum_{k \in K} \phi_{a k} t_{a}(k) y_{a}(k)=\sum_{k \in K} \phi_{a}\left(L_{a} / t_{a}(k)\right) t_{a}(k)\left[U_{a}(k)-U_{a}(k-1)\right] .
$$

Assumption 2. The emission rate function $\phi_{a}(\cdot)$ is continuous with respect to vehicle speed.

Proposition 1. Under Assumptions 1 and 2, the link emissions obtained from Eq. (13) converge to the continuous-time link emissions obtained from Eq. (2) when the length of interval $\delta$ approaches 0.

The proof is presented in Appendix B.

\subsubsection{Estimate link emissions from sub-packets}

As stated above, the sub-packet $y_{a}(k, l)$ has an estimated travel time of $(l-k) \delta$. The average speed of vehicles in this sub-packet can be estimated as follows:

$$
S_{a k l}=\frac{L_{a}}{(l-k) \delta} .
$$

The average vehicle speed can be used to estimate the emission rate of sub-packet $y_{a}(k, l)$, as follows:

$$
\phi_{a k l}=\phi_{a}\left(s_{a k l}\right) .
$$

We estimate the total emissions of the packet $y_{a}(k)$ by summing the total emissions of each sub-packet, i.e., $\sum_{l \in K} \phi_{a}\left(s_{a k l}\right)(l-k) \delta y_{a}(k, l)$. We estimate the total emissions arising from travelers using link $a$ during the period under study by summing the total emissions of individual packets, as follows:

$$
\tilde{E}_{a}=\sum_{k \in K} \sum_{l \in K} \tilde{\phi}_{a k l} y_{a}(k, l)=\sum_{k \in K} \sum_{l \in K} \tilde{\phi}_{a k l}\left[Y_{a}(k, l)-Y_{a}(k, l-1)\right] .
$$

where $\tilde{\phi}_{a k l}$ is a positive constant and

$$
\tilde{\phi}_{a k l}=\phi_{a k l}(l-k) \delta=\phi_{a}\left(s_{a k l}\right)(l-k) \delta=\phi_{a}\left(L_{a} /(l-k) \delta\right)(l-k) \delta
$$

Proposition 2. Under Assumptions 1 and 2, the link emissions obtained from Eq. (16) converge to the link emissions obtained from Eq. (13) and the continuous-time link emissions obtained from (2) when the length of interval $\delta$ approaches 0 .

The proof is presented in Appendix C.

\section{LTM-based SO-DTA problems with environmental objectives}

\subsection{Notations}

We consider a network $G(N, A)$ with multiple origins and a single destination. $N$ and $A$ denote sets of nodes and arcs (links), respectively. The links in the network are classified into three 
categories: source links, destination links, and general links. It is assumed that each source (destination) link is connected to a single origin (destination) node, and that each source (destination) node is connected to a single origin (destination) link. The source and destination links are both dummy links. All of the source and destination links have an infinite inflow capacity and an infinite storage capacity, and vehicles remain at destination links on arrival. As the network has a single destination, there is only one destination link. The following additional notation is used throughout the paper:

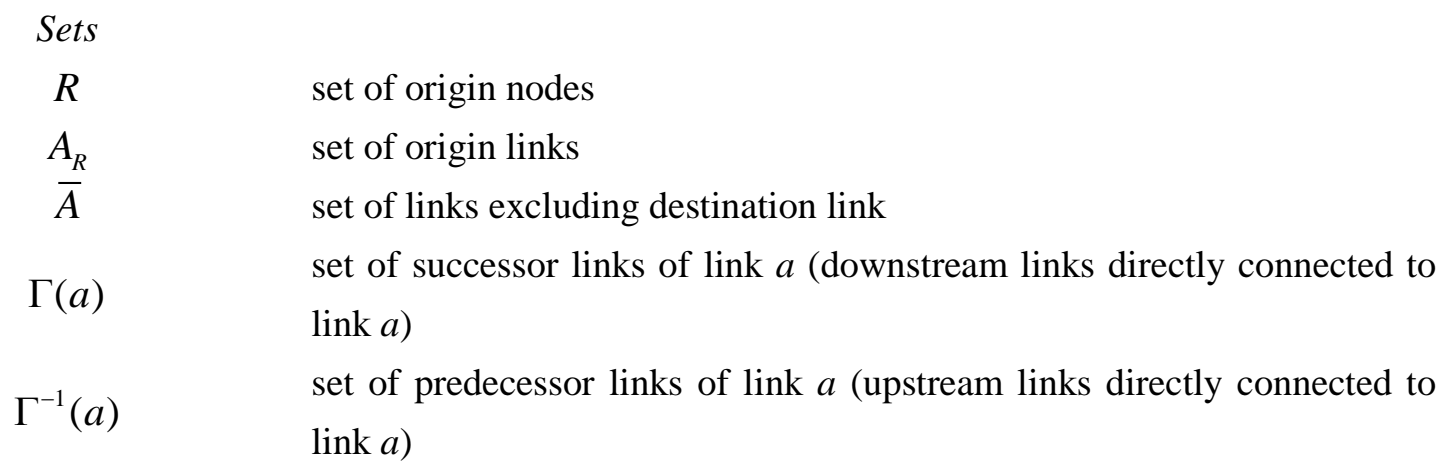

\section{Parameters}

$v_{a} \quad$ free flow speed of vehicles on link $a$

$w_{a} \quad$ backward shock-wave speed of traffic on link $a$

$\bar{l}_{a}$ travel time required by the backward shock-wave from the exit to the entrance of link $a$

$\rho_{\text {jam }} \quad$ jam density

$Q_{a}(k) \quad$ link inflow capacity during interval $k$

$C_{a}(k) \quad$ link outflow capacity during interval $k$

$D_{a}(k) \quad$ of interval $k$

Variables

$W_{a b}(k)$

cumulative number of vehicles that leave link $a$ and enter link $b$ by the

Vectors

$\begin{array}{ll}\mathbf{U} & {\left[U_{a}(k), a \in A, k \in K\right]} \\ \mathbf{V} & {\left[V_{a}(k), a \in \bar{A}, k \in K\right]} \\ \mathbf{W} & {\left[W_{a b}(k), a \in \bar{A}, b \in \Gamma(a), k \in K\right]} \\ \mathbf{Y} & {\left[Y_{a}(k, l), a \in \bar{A}, k \in K, l \in K\right]} \\ \mathbf{X} & \mathbf{x}=[\mathbf{U}, \mathbf{V}, \mathbf{W}]\end{array}$

Acronyms of SO-DTA problems

TSTT-SO-DTA SO-DTA problem with efficiency objective and without NVH constraints

TSTT-NVH-SO-DTA SO-DTA problem with efficiency objective and NVH constraints

TSE-SO-DTA SO-DTA problem with environmental objective and without NVH constraints 
TSE-NVH-SO-DTA SO-DTA problem with environmental objective and NVH constraints

TSTT-TSE-SO-DTA SO-DTA problem with efficiency and environmental objectives and without NVH constraints

TSTT-TSE-NVH-SO-DTA SO-DTA problem with efficiency and environmental objectives and NVH constraints

\subsection{Overview of LTM dynamic network constraints}

The LTM is based on a triangular fundamental diagram (Yperman, 2007) and defined by three parameters: fixed free-flow speed $(v)$, maximum flow or capacity $\left(q_{\max }\right)$, and jam density ( $\left.\rho_{\text {jam }}\right)$ (see Fig. 3). Both critical density, $\rho_{\text {crit }}$, and backward shock wave speed, $w$, can be derived from these three parameters, as follows: $\rho_{\text {crit }}=q_{\max } / v$ and $w=q_{\max } v /\left(q_{\max }-\rho_{\text {jam }} v\right)$. Newell's simplified method is used to determine sending and receiving flow.

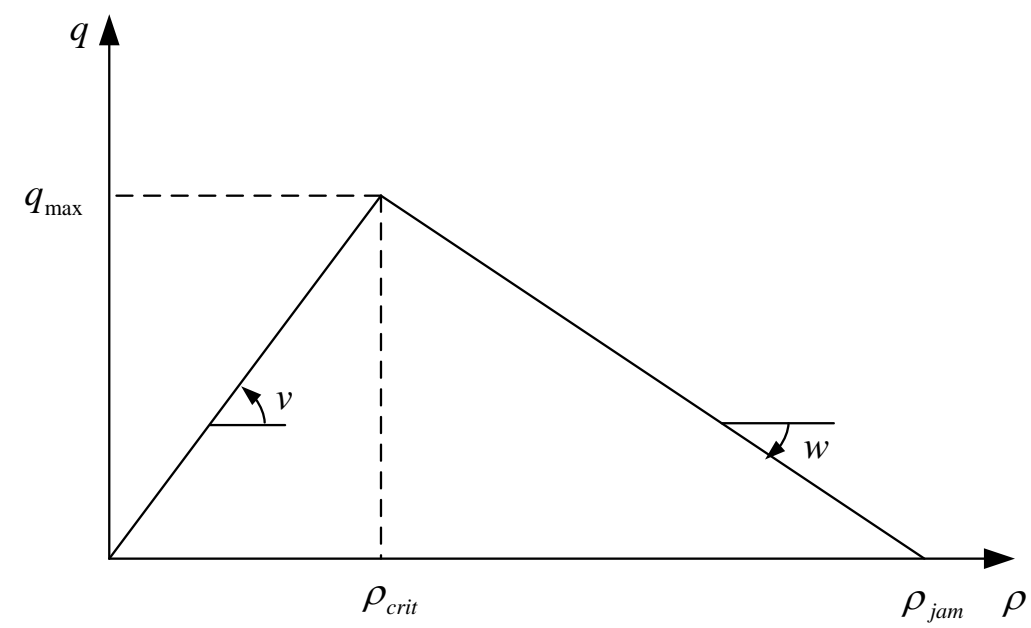

Fig. 3. Triangular fundamental diagram (Yperman, 2007).

The sending flow of a link is constrained by both the boundary conditions at the upstream end of the link and the outflow capacity of the link. According to Newell's (1993) simplified theory, a free-flow traffic state observed at the downstream link boundary at the end of interval $k$ must have been emitted from the upstream boundary $L_{a} / \delta v_{a}$ time units earlier (i.e., with a free-flow travel time of $\vec{\imath}_{a}$ ). The sending flow of link $a$ during interval $k$ can be mathematically expressed as follows (Yperman, 2007):

$$
S_{a}(k)=\min \left\{U_{a}\left(k-\vec{l}_{a}\right)-V_{a}(k-1), C_{a}(k)\right\} .
$$

The receiving flow of a link is constrained by both the boundary conditions at the downstream end of the link and the inflow capacity of the link. According to Newell's (1993) simplified theory, a congested traffic state observed at the upstream boundary at the end of interval $k$ must have been emitted from the downstream boundary $-L_{a} / \delta w_{a}$ time units earlier (i.e., with a backward shock wave travel time of $\overline{i_{a}}$ ), as congested traffic travels at a (negative) speed of $w_{a}$. The receiving flow of link $a$ during interval $k$ can be mathematically expressed as follows (Yperman, 2007): 


$$
R_{a}(k)=\min \left\{V_{a}\left(k-\bar{i}_{a}\right)+L_{a} \rho_{j a m}-U_{a}(k-1), Q_{a}(k)\right\} .
$$

The outflow of an ordinary link $a$ with only one successor link $b$ can be obtained as follows:

$$
V_{a}(k)-V_{a}(k-1)=\min \left\{S_{a}(k), R_{b}(k)\right\} .
$$

In the LTM, Eq. (19) is a disjunctive function, and its feasible region contains a non-convex set, as in the CTM (Zhang and Chiu, 2011). Similar to the CTM-based SO-DTA model reported by Ziliaskopoulos (2000), the LTM-based SO-DTA model enables Eqs. (17)-(19) to be relaxed into the following system of linear LTM-based flow constraints (Long and Szeto, 2015):

$$
\left\{\begin{array}{l}
V_{a}(k) \leq U_{a}\left(k-\vec{\imath}_{a}\right), \forall a \in \bar{A}, k \in K, \\
V_{a}(k)-V_{a}(k-1) \leq C_{a}(k), \forall a \in \bar{A}, k \in K, \\
U_{b}(k) \leq V_{b}\left(k-\bar{\imath}_{b}\right)+L_{b} \rho_{j a m}, \forall a \in \bar{A}, b \in \Gamma(a), k \in K, \\
U_{b}(k)-U_{b}(k-1) \leq Q_{b}(k), \forall a \in \bar{A}, b \in \Gamma(a), k \in K .
\end{array}\right.
$$

The LTM also requires traffic flow to satisfy flow conservation and definitional constraints. Under flow conservation constraints, all traffic entering a node (except a destination node), together with the demand generated at this node, must exit from the node. We use the following flow conservation constraints:

$$
\begin{aligned}
& U_{a}(k)=D_{a}(k), \forall a \in A_{R}, k \in K, \\
& U_{a}(k)=\sum_{b \in \Gamma^{-1}(a)} W_{b a}(k), \forall a \in A \backslash A_{R}, k \in K, \text { and } \\
& V_{a}(k)=\sum_{b \in \Gamma(a)} W_{a b}(k), \forall a \in \bar{A}, k \in K .
\end{aligned}
$$

In addition, all vehicles should arrive at their destinations during the studied period. Equivalently, beside the destination link, the number of vehicles on all links should be zero at the end of the studied period. Hence, we have

$$
\sum_{a \in \bar{A}}\left[U_{a}(\underline{K})-V_{a}(\underline{K})\right]=0,
$$

Definitional constraints are used to describe the initial conditions and the non-negative and no n-decreasing properties of the cumulative flow. We use the following constraints:

$$
\begin{aligned}
& W_{a b}(k)-W_{a b}(k-1) \geq 0, \forall a \in \bar{A}, b \in \Gamma(a), k \in K, \text { and } \\
& Y_{a b}(0)=0, \forall a \in \bar{A}, b \in \Gamma(a) .
\end{aligned}
$$

Constraints (25) and (26) imply that the cumulative transfer flow is non-negative. Constraints (22)-(26) imply that the cumulative link inflow and outflow are non-decreasing, non-negative, and are initially equal to 0.Definition 1 (basic feasible flow set). Constraints (20)-(26) form a basic feasible flow set for an LTM-based SO-DTA problem in a network with a single destination . The set is formulated as follows: 


$$
\Omega=\{\mathbf{x} \mid \text { Subject to constraints (20)-(26) }\}
$$

\subsection{NVH constraints}

In the LTM-based SO-DTA model, constraint (18) in the LTM is relaxed to constraint (20), which is a set of "less than or equal to" constraints. An optimal solution to the SO-DTA model is obtained if a link-interval pair exists such that all of the constraints in condition (20) fall into the inequality region. As a result, vehicles are likely to be held in the link without moving forward, even if there is enough capacity in the successor link. This solution property is known as VH (Zheng and Chiu, 2011). Therefore, $\mathrm{VH}$ phenomenon can be avoided such that at least one of the following less than or equal to inequalities is equal for each $a \in \bar{A}$ and $k \in K$ (Long and Szeto, 2015):

$$
\left\{\begin{array}{l}
V_{a}(k) \leq U_{a}\left(k-\vec{\imath}_{a}\right), \\
V_{a}(k)-V_{a}(k-1) \leq C_{a}(k), \\
U_{b}(k) \leq V_{b}\left(k-\overleftarrow{\imath}_{b}\right)+L_{b} \rho_{j a m}, \forall b \in \Gamma(a), \\
U_{b}(k)-U_{b}(k-1) \leq Q_{b}(k), \forall b \in \Gamma(a) .
\end{array}\right.
$$

For any non-destination link $a$ with $|\Gamma(a)|$ successor links, the first two inequalities in system (28) restrict the sending flow of link $a$, and the last two inequalities in system (28) restrict the receiving flow of all of link $a$ 's successor links. Therefore, the number of constraints in system (28) with respect to interval $k$ is $2+2|\Gamma(a)|$. The $\mathrm{NVH}$ conditions require that an equality holds for at least one of the $2+2|\Gamma(a)|$ constraints. Accordingly, the NVH conditions can be formulated as the following mixed-integer constraints (Long and Szeto, 2015):

$$
\begin{gathered}
-\left[\theta_{a}^{0}(k)+\sum_{i=1}^{m_{a}} \theta_{a}^{i}(k)\right] M \leq V_{a}(k)-U_{a}\left(k-\vec{\imath}_{a}\right), \forall a \in \bar{A}, k \in K, \\
-\left[1-\theta_{a}^{0}(k)+\sum_{i=1}^{m_{a}} \theta_{a}^{i}(k)\right] M \leq V_{a}(k)-V_{a}(k-1)-C_{a}(k), \forall a \in \bar{A}, k \in K, \\
-\left[\sum_{i=1}^{m_{a}} \sigma_{i}^{j}+\theta_{a}^{0}(k)-\sum_{i=1}^{m_{a}}\left(2 \sigma_{i}^{j}-1\right) \theta_{a}^{i}(k)\right] M \leq U_{b_{j}}(k)-V_{b_{j}}\left(k-\bar{t}_{b_{j}}\right)-L_{b_{j}} \rho_{j a m},, \forall a \in \bar{A}, k \in K, j \in J_{a}, \\
-\left[1+\sum_{i=1}^{m_{a}} \sigma_{i}^{j}-\theta_{a}^{0}(k)-\sum_{i=1}^{m_{a}}\left(2 \sigma_{i}^{j}-1\right) \theta_{a}^{i}(k)\right] M \leq U_{b_{j}}(k)-U_{b_{j}}(k-1)-Q_{b_{j}}(k), \forall a \in \bar{A}, k \in K, j \in J_{a}, \\
\sum_{i=0}^{m_{a}} 2^{i} \theta_{a}^{i}(k) \leq 1+2|\Gamma(a)|, \forall a \in \bar{A}, k \in K, \\
\theta_{a}^{i}(k) \in\{0,1\}, \forall, \forall a \in \bar{A}, k \in K, i=0,1, \cdots, m_{a} .
\end{gathered}
$$

where $M$ is a very large positive value, $J_{a}=\{1,2, \cdots,|\Gamma(a)|\}$ is an index set for link $a$ 's successor links, $b_{j}$ is the $j$ th link in $\Gamma(a), m_{a}=\arg \min _{m}\left\{2^{m+1} \geq 2+2|\Gamma(a)|\right\}$, and $\sigma_{i}^{j}$ is 0 or 1 , such that 
$\sum_{i=1}^{m_{a}} 2^{i-1} \sigma_{j}^{i}=j$. System (29) contains $m_{a}+1$ integer variables, which can form $2^{m_{a}+1}$ combinations. System (28) contains $2+2|\Gamma(a)|$ LTM-based flow constraints, and constraint (29g) implies that only $2+2|\Gamma(a)|$ combinations of the integer variables can be used.

\subsection{Link inflow decomposition constraints}

As link emissions are estimated using decomposed link inflow, link inflow decomposition constraints are developed to formulate a relationship between cumulative link sub-flow and cumulative link inflow and outflow. As shown in Fig. 2, Eq. (7) can also be formulated as follows:

$$
Y_{a}(k, l)= \begin{cases}0, & \text { if } V_{a}(l) \leq U_{a}(k-1), \\ V_{a}(l)-U_{a}(k-1), & \text { if } U_{a}(k-1)<V_{a}(l)<U_{a}(k), \forall a \in \bar{A}, k \in K, l \in K . \\ U_{a}(k)-U_{a}(k-1), & \text { if } V_{a}(l) \geq U_{a}(k) .\end{cases}
$$

We offer the following proposition:

Proposition 3. For any given vectors $\mathbf{U}$ and $\mathbf{V}$, vector $\mathbf{Y}$ satisfies Eq. (30) if and only if it fulfills the following conditions:

$$
\begin{aligned}
& Y_{a}(k, l) \geq 0, \forall a \in \bar{A}, k \in K, l \in K, \\
& Y_{a}(k, l) \leq U_{a}(k)-U_{a}(k-1), \forall a \in \bar{A}, k \in K, l \in K, \\
& \sum_{k} Y_{a}(k, l)=V_{a}(l), \forall a \in \bar{A}, l \in K, \\
& Y_{a}(k, l)=0, \text { if } V_{a}(l) \leq U_{a}(k-1), \forall a \in \bar{A}, k \in K, l \in K, \text { and } \\
& Y_{a}(k, l)=U_{a}(k)-U_{a}(k-1), \text { if } V_{a}(l) \geq U_{a}(k), \forall a \in \bar{A}, k \in K, l \in K .
\end{aligned}
$$

The proof is presented in Appendix D.

The conditional constraints (34) and (35) can be reformulated within the mixed integer programming framework using the well-known "Big M" method, which introduces a large positive coefficient, $M$. We offer the following proposition:

Proposition 4. Constraints (31)-(35) can be equivalently formulated as follows:

$$
\begin{aligned}
& Y_{a}(k, l) \geq 0, \forall a \in \bar{A}, k \in K, l \in K, \\
& Y_{a}(k, l) \leq U_{a}(k)-U_{a}(k-1), \forall a \in \bar{A}, k \in K, l \in K, \\
& \sum_{k \in K} Y_{a}(k, l)=V_{a}(l), \forall a \in \bar{A}, l \in K, \\
& Y_{a}(k, l) \leq \varpi_{a}(k-1, l) M, \forall a \in \bar{A}, k \in K, l \in K, \\
& Y_{a}(k, l) \geq U_{a}(k)-U_{a}(k-1)-\left[1-\varpi_{a}(k-1, l)\right] M, \forall a \in \bar{A}, k \in K, l \in K,
\end{aligned}
$$




$$
\begin{aligned}
& V_{a}(l)-U_{a}(k) \leq \varpi_{a}(k, l) M, \forall a \in \bar{A}, k \in K, l \in K, \\
& V_{a}(l)-U_{a}(k) \geq\left[\varpi_{a}(k, l)-1\right] M, \forall a \in \bar{A}, k \in K, l \in K, \\
& \varpi_{a}(k, l) \in\{0,1\}, \forall a \in \bar{A}, k \in K, l \in K .
\end{aligned}
$$

The proof is presented in Appendix E.

\subsection{Overview of SO-DTA models in terms of TSTT}

The TSTT for the whole network is the sum of link travel times of all vehicles in the network, and can be formulated as follows:

$$
T S T T=\sum_{a \in \bar{A}} \sum_{k \in K} y_{a}(k) t_{a}(k) .
$$

Assumption 3. All vehicles arrive at their destinations during the studied period.

Proposition 5: Under Assumption 3, Eq. (37) can be equivalently formulated as follows:

$$
T S T T=\delta \sum_{a \in \bar{A}} \sum_{k \in K}\left[U_{a}(k)-V_{a}(k)\right] .
$$

The proof is presented in Appendix F.

Because $U_{a}(k)-V_{a}(k)$ is the number of vehicles on link $a$ at the end of interval $k$, the TSTT obtained from Eq. (38) is consistent with Ziliaskopoulos' (2000) formulation. The objective of an LTM-based TSTT-SO-DTA model is to minimize TSTT in a network, which equals the sum of the number of vehicles on all links within the modeling horizon. Therefore, a TSTT-SO-DTA problem can be formulated as the following LP problem (Long and Szeto, 2015):

$$
\min _{\mathbf{x} \in \Omega} T S T T=\delta \sum_{a \in \bar{A}} \sum_{k \in K}\left[U_{a}(k)-V_{a}(k)\right] .
$$

According to Long and Szeto (2015), a TSTT-NVH-SO-DTA problem can be formulated as the following LP problem:

$$
\max _{\mathbf{x} \in \Omega} \tilde{\varpi}=\sum_{a \in A \backslash \bar{A}} \sum_{k \in K} U_{a}(k)+\alpha \sum_{a \in \bar{A}} \sum_{k \in K} V_{a}(k),
$$

where $\alpha$ is a sufficiently small positive constant. The first term on the right hand side of Eq. (40) is to achieve an earliest arrival flow (EAF) pattern. According to Zheng and Chiu (2011), the R-SO-DTA problem is equivalent to the EAF problem. The second term on the right hand side of Eq. (40) is a penalty term. Long and Szeto (2015) proved that if the positive constant $\alpha$ is sufficiently small, the optimal solution of LP problem (40) is an NVH-SO flow pattern in terms of the TSTT.

\subsection{SO-DTA models with environmental objectives}

\subsubsection{TSE-SO-DTA problems}

The TSE of the whole network is estimated by summing the emissions from all of the links in the 
network. Either Eq. (13) or Eq. (16) below can be used to compute TSE:

$$
\begin{aligned}
& T S E_{1}=\sum_{a \in \bar{A}} \sum_{k \in K} \phi_{a}\left(L_{a} / t_{a}(k)\right) t_{a}(k)\left[U_{a}(k)-U_{a}(k-1)\right], \text { and } \\
& T S E_{2}=\sum_{a \in \bar{A}} \sum_{k \in K} \sum_{l \in K} \tilde{\phi}_{a k l} y_{a}(k, l)=\sum_{a \in \bar{A}} \sum_{k \in K} \sum_{l \in K} \tilde{\phi}_{a k l}\left[Y_{a}(k, l)-Y_{a}(k, l-1)\right] .
\end{aligned}
$$

According to Eq. (10), $t_{a}(k)$ is a nonlinear and nonconvex function with respect to link cumulative flows. If Eq. (41) is used, TSE is a non-linear and non-convex function with respect to cumulative link flow (comprising cumulative link inflow, outflow, and sub-flow). In contrast, Eq. (42) gives a linear TSE function with respect to cumulative link flow. Therefore, we use the latter as the objective function for the TSE-SO-DTA problem. Combining this objective function with LTM-based dynamic network constraints and link inflow decomposition constraints, we formulate the TSE-SO-DTA problem as the following MILP problem:

$$
\min _{\mathbf{x} \in \Omega, \mathbf{Y}, \mathbf{\omega}} \operatorname{TSE}=\sum_{a \in A} \sum_{k \in K} \sum_{l \in K} \tilde{\phi}_{a k l}\left[Y_{a}(k, l)-Y_{a}(k, l-1)\right],
$$

Subject to Constraints (36),

where $\boldsymbol{\omega}=\left[\varpi_{a}(k, l)\right]$.

\subsubsection{TSE-NVH-SO-DTA problem}

If NVH constraints are also considered, the TSE-NVH-SO-DTA problem can be formulated as the following MILP problem:

$$
\min _{\mathbf{x} \in \Omega, \mathbf{Y}, \boldsymbol{\theta ,}, \mathbf{\omega}} T S E=\sum_{a \in \bar{A}} \sum_{k \in K} \sum_{l \in K} \tilde{\phi}_{a k l}\left[Y_{a}(k, l)-Y_{a}(k, l-1)\right],
$$

Subject to Constraints (29) and (36),

where $\boldsymbol{\theta}=\left[\theta_{a}^{i}(k)\right]$.

\subsubsection{The TSTT-TSE-SO-DTA problem}

The TSTT-TSE-SO-DTA problem aims to predict an optimal time-dependent traffic state that simultaneously minimizes network-wide congestion and vehicle emissions for the whole network. The TSTT-TSE-SO-DTA problem can be formulated as a bi-objective MILP problem:

$$
\min _{\mathbf{x} \in \Omega, \mathbf{Y}, \boldsymbol{\omega}}[T S T T, T S E]=\left[\delta \sum_{a \in \bar{A}} \sum_{k \in K}\left[U_{a}(k)-V_{a}(k)\right], \sum_{a \in A} \sum_{k \in K} \sum_{l \in K} \tilde{\phi}_{a k l}\left[Y_{a}(k, l)-Y_{a}(k, l-1)\right]\right],
$$

Subject to Constraint (36).

\subsubsection{The TSTT-TSE-NVH-SO-DTA problem}

If NVH constraints are also considered, the TSTT-TSE-NVH-SO-DTA problem can be formulated as the following bi-objective MILP problem: 


$$
\min _{\mathbf{x} \in \Omega, \mathbf{Y}, \boldsymbol{\theta}, \boldsymbol{\omega}}[T S T T, T S E]=\left[\delta \sum_{a \in \bar{A}} \sum_{k \in K}\left[U_{a}(k)-V_{a}(k)\right], \sum_{a \in \bar{A}} \sum_{k \in K} \sum_{l \in K} \tilde{\phi}_{a k l}\left[Y_{a}(k, l)-Y_{a}(k, l-1)\right]\right],
$$

Subject to Constraints (29) and (36).

\section{Numerical examples}

In this section, four numerical examples are presented to illustrate the performance of the proposed models. In the first example, a single link network is used to evaluate the accuracy of the proposed method to estimate TSEs. The second example uses a merged network to demonstrate better modeling accuracy of the proposed SO-DTA model than existing models. The third example uses Ziliaskopoulos's (2000) network to compare the optimal solutions provided by various SO-DTA models. The last example uses the modified Nguyen and Dupuis (1984) network to demonstrate that the proposed LTM-based models are more computational efficiency than the CTM-based models. All of the experiments are run on a computer with an Intel ${ }^{\circledR}$ Core $^{\mathrm{TM}} 2$ Quad Q9550 $2.83 \mathrm{GHz}$ central processing unit and 3.5 GB random-access memory. All of the LP and MILP problems are solved using the commercial software package Gurobi (version 6.0).

In all experiments, we focus on $\mathrm{CO}$ emissions, and use the following $\mathrm{CO}$ emission rate function (Stein and Walker, 2003; Aziz and Ukkusuri, 2012):

$$
\phi(V)=-0.064+0.0056 V+0.00026(V-50)^{2},
$$

where $\phi(V)$ is the emission rate function (in grams of CO per vehicle per second) and $V$ is the speed of the vehicle in miles per hour (mph).

Example 1. A single link network: evaluating the accuracy of the estimated TSEs.

In this example, we adopted a network with single link to evaluate the accuracy of the TSEs obtained from Eqs. (13) and (16), respectively. We assumed that the link is empty initially. The input parameters for the LTM are given as follows: 133 vehicles (veh)/km for jam density, $20 \mathrm{~m} / \mathrm{s}$ for free flow speed, $10 \mathrm{~m} / \mathrm{s}$ for backward shockwave speed; $36 \mathrm{veh} / \mathrm{min}$ for flow capacity, and $2.4 \mathrm{~km}$ for link length. Following Carey and Ge (2005) and Long et al (2011), the traffic demand lasts 24 min, and the following demand function is adopted:

$$
d(t)= \begin{cases}32 \sin (\pi t / 10), & \text { if } 0 \leq t<5, \\ 32, & \text { if } 5 \leq t<10, \\ 20+12 \sin ^{5}(\pi(t+4) / 28), & \text { if } 10 \leq t \leq 24,\end{cases}
$$

where $d(t)$ is the demand rate at time $t$ in veh/min. We set a constant outflow capacity at 25.2 veh/min.

We set a very short interval length (0.01 seconds), use the LTM to obtain the cumulative-flow curves for the links, and obtain the theoretical value of TSE using Eq. (13), which gives $30810.76 \mathrm{gm}$. 
The theoretical value of TSE offers a very useful means of evaluating the accuracy of the proposed methods of estimating TSE. We vary the length of the interval from 0.1 seconds to 20 seconds, generate cumulative flow curves from the LTM, and use the two proposed methods to estimate TSE for different interval lengths. The relative error of the two TSE-estimation methods is provided in Table 1. Both methods exhibit a very high accuracy, and relative estimation error increases uniformly for both methods with an increase in $\delta$. These findings indicate that shortening interval length can improve the accuracy of TSE estimation. We also observe from the table that TSE is slightly under-estimated using Eq. (13) and slightly over-estimated using Eq. (16).

Table 1. Relative error of TSE estimation.

\begin{tabular}{|c|c|c|c|c|c|}
\hline Interval length $\delta(\mathrm{s})$ & 0.1 & 1 & 5 & 10 & 20 \\
\hline$T_{S E}$ (g) & 30810.76 & 30810.73 & 30809.12 & 30806.2 & 30789.07 \\
\hline Error of TSE $E_{1}(\%)$ & 0.00 & $-9.74 \mathrm{E}-05$ & $-5.32 \mathrm{E}-03$ & $-1.48 \mathrm{E}-02$ & $-7.04 \mathrm{E}-02$ \\
\hline$T S E_{2} \quad(\mathrm{~g})$ & 30810.76 & 30810.85 & 30812.05 & 30817.63 & 30835.53 \\
\hline Error of $T^{2} E_{2}(\%)$ & 0.00 & $2.92 \mathrm{E}-04$ & 4.19E-03 & $2.23 \mathrm{E}-02$ & 8.04E-02 \\
\hline
\end{tabular}

Example 2. TSE-SO-DTA problem in a merged network: comparing the performance of the proposed model with existing models.

In this example, a merged network (see Fig. 4) consisting of six nodes, five links, and two origin-destination (OD) pairs (from Nodes $r_{1}$ and $r_{2}$ to Node $s$ ) is considered. The length of each link is the same and is $200 \mathrm{~m}$. The interval length is $\delta=10 \mathrm{~s}$. Both the free flow and backward shockwave speeds are $72 \mathrm{~km} / \mathrm{h}$ (i.e., $20 \mathrm{~m} / \mathrm{s}$ ). Hence, the free-flow travel times on all links are one interval long, and the LTM is equivalent to the CTM in this example. The initial state of the network is empty. Table 2 provides the maximum occupancy and flow capacity of each link. The flow capacity is 20 veh/interval for Links 1, 2, and 5, and 12 veh/interval for other two links. The traffic demands of both OD pairs are the same. To demonstrate the accuracy of the proposed TSE-SO-DTA model, we construct three scenarios with different levels of traffic demands. The traffic demand in the three scenarios comprises 3,6 , and 16 vehicles for each OD pair, respectively, during the first three intervals, and 0 vehicles during the other intervals.

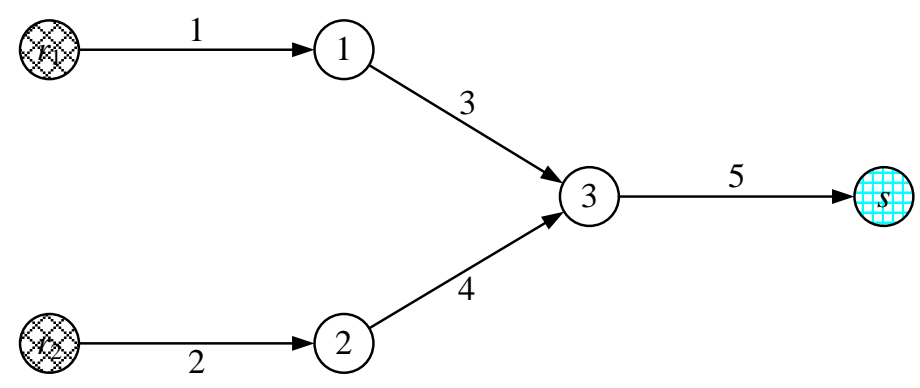


Fig. 4. A merge network for Example 2.

Table 2. Setting for the merge network.

\begin{tabular}{llllll}
\hline Link & 1 & 2 & 3 & 4 & 5 \\
\hline$N_{a}$ & 100 & 100 & 20 & 20 & 100 \\
$C_{a}$ & 20 & 20 & 12 & 12 & 20 \\
\hline
\end{tabular}

In this example, we compared our LTM-based model (i.e., proposed model (43)) with the CTM-based model with approximation proposed by Aziz and Ukkusuri (2012). In our model, the emission rate is estimated by the speed deduced from average link travel times. However, in the models proposed by Aziz and Ukkusuri (2012), the emission rate is estimated by vehicle instantaneous speed of each cell, and the obtained TSE from this emission rate (denoted as $T_{S E}$ ) is a nonlinear nonconvex function with respect to cell flows. As a result, the SO-DTA model taking $T S E_{3}$ as the objective in the study of Aziz and Ukkusuri (2012) is nonlinear and nonconvex mathematical program. Aziz and Ukkusuri (2012) proposed a formulation to approximate TSE $E_{3}$. The approximated TSE (denoted as $T S E_{4}$ ) is a quadratic function of cell flows, and the SO-DTA model taking $T S E_{4}$ as the objective in the study of Aziz and Ukkusuri (2012) is a QP model.

Both the proposed model (43) in this paper and the QP model proposed by Aziz and Ukkusuri (2012) were used to solve the TSE-SO-DTA problems in this example. The TSEs corresponding to the optimal solutions of each model are provided in Table 3, where $T S E_{1}$ and $T S E_{2}$ were obtained by Eqs. (41) and (42), respectively; and TSE $E_{3}$ and $T S E_{4}$ were obtained by Eqs. (6) and (7) in the study of Aziz and Ukkusuri (2012), respectively. One can observe from Table 3 that $T S E_{1}, T S E_{2}$, and $T S E_{3}$ have very close values for all scenarios. In contrast, the value of $T S E_{4}$ is obviously less than the values of $T S E_{1}, T S E_{2}$, and $T S E_{3}$, and even can be negative when traffic demand is light. This implies that the approximation based on $T S E_{4}$ can be very inaccurate. In addition, one can also observe from Table 3 that all the values of $T S E_{1}, T S E_{2}$, and $T S E_{3}$ obtained from the optimal solutions to the proposed model are less than the TSEs obtained from the corresponding optimal solution to the QP model proposed by Aziz and Ukkusuri (2012). This implies that proposed model outperforms their QP model in terms of obtaining lower TSEs for TSE-SO-DTA problems.

Table 3. Comparison of TSE values obtained from different models and estimation methods.

\begin{tabular}{ccrrrr}
\hline Scenario & Model & $T S E_{1}(\mathrm{~g})$ & $T S E_{2}(\mathrm{~g})$ & $T S E_{3}(\mathrm{~g})$ & $T S E_{4}(\mathrm{~g})$ \\
\hline \multirow{2}{*}{ Scenario 1 } & This paper & 232.48 & 232.48 & 232.48 & -17.31 \\
& Aziz and Ukkusuri & 239.69 & 247.08 & 240.64 & -26.59 \\
\hline \multirow{2}{*}{ Scenario 2 } & This paper & 563.40 & 594.13 & 573.19 & 354.67 \\
& Aziz and Ukkusuri & 568.23 & 594.13 & 575.05 & 353.01 \\
\hline \multirow{2}{*}{ Scenario 3 } & This paper & 1171.27 & 1198.85 & 1200.70 & 1050.15
\end{tabular}


Example 3. SO-DTA problems in Ziliaskopoulos's (2000) network: comparing the optimal solutions provided by various SO-DTA models.

The test network shown in Fig. 5 is derived from Ziliaskopoulos (2000) and contains 9 nodes, 10 links, and 1 OD pair (from Node $r$ to Node $s$ ). Same as Example 2, interval length is $10 \mathrm{~s}$; the length of each link is $200 \mathrm{~m}$; the free flow and backward shockwave speeds are $72 \mathrm{~km} / \mathrm{h}$ (i.e., $20 \mathrm{~m} / \mathrm{s}$ ). The initial state of the network is empty. Table 4 provides the maximum occupancy and flow capacity of each link. The flow capacity is 12 veh/interval for Links 2 and 6 veh/interval for other links except Link 4. Following Ziliaskopoulos (2000), we assumed an accident occurred on Link 4 and that the flow capacity of Link 4 is thus time-varying: 0 veh/interval for the first four time intervals; 3 veh/interval for time intervals 5 and 6 ; and 6 veh/interval for the remaining time intervals. The traffic demands are eight vehicles for intervals 1 and 3, 16 for interval 2, and 0 vehicle for the rest of intervals. The parameter for LP problem (40) is $\alpha=0.0001$.

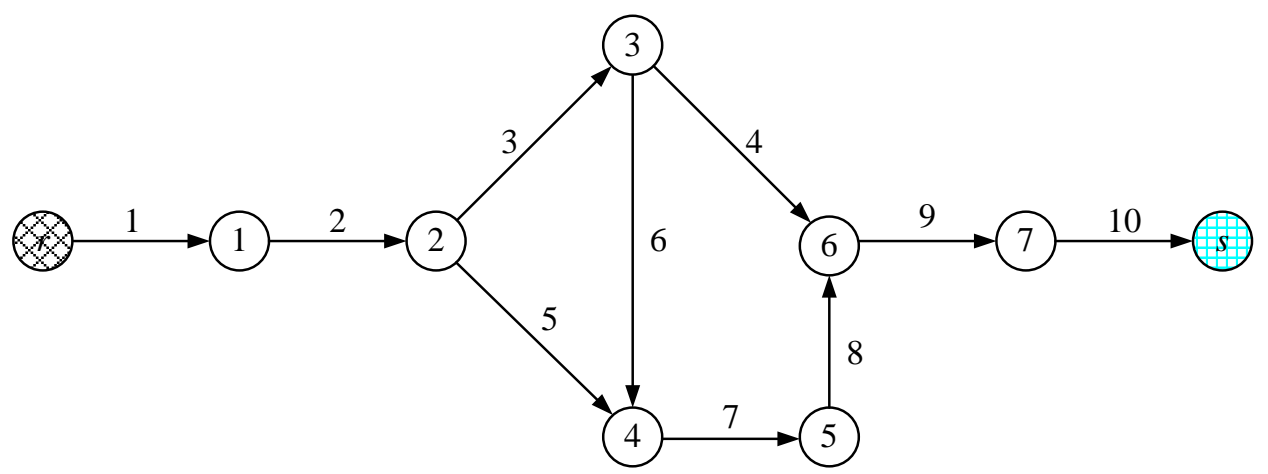

Fig. 5. The Ziliaskopoulos (2000) network for Example 3.

Table 4. Setting for the Ziliaskopoulos (2000) network.

\begin{tabular}{ccccccccccc}
\hline Link & 1 & 2 & 3 & 4 & 5 & 6 & 7 & 8 & 9 & 10 \\
\hline$N_{a}$ & 100 & 20 & 10 & 10 & 10 & 10 & 10 & 10 & 20 & 100 \\
$C_{a}$ & 12 & 12 & 6 & - & 6 & 6 & 6 & 6 & 12 & 12 \\
\hline
\end{tabular}

We firstly solved the TSTT-, TSTT-NVH-, TSE-, and TSE-NVH-SO-DTA problems, and calculated the TSTTs and TSEs corresponding to their optimal solutions, which are provided in rows 3 and 4 of Table 5. One can observe that the SO-DTA problems either considering or not considering NVH constraints can have the same objectives. We fixed the TSTT (TSE) at the minimal TSTT (TSE), and solved the revised SO-DTA models that minimize or maximize TSE (TSTT). The TSTTs and TSEs corresponding to the optimal solutions to those revised SO-DTA problems are provided in the last four rows of Table 5. One can observe that the values of minimum and maximum TSE (TSTT) are 
different when the value of TSTT (TSE) is fixed at the minimal TSTT (TSE). For example, the values of TSE can be varied from $450.86 \mathrm{~g}$ to $482.70 \mathrm{~g}$ when the TSTT is restricted to be its minimum value (i.e., 215 intervals), and the values of TSTT can be varied from 218 intervals to 221 intervals when TSE is restricted to be its minimum value (i.e., $446.90 \mathrm{~g}$ ). This implies that the TSTT-, TSTT-NVH-, TSE-, and TSE-NVH-SO-DTA problems can have multiple optimal solutions.

We further solved the bi-objective SO-DTA models (45) and (46), and graphically showed the Pareto frontiers of the two models in Fig. 6. One can clearly observe a trade-off between improving traffic efficiency (i.e., lowering TSTT) and mitigating traffic-induced environment pollution (i.e., lowering TSE). One can also observe that the Pareto frontier moves away from the origin when NVH constraints are considered, and the Pareto frontier of the TSTT-TSE-NVH-SO-DTA model can be discontinuous. The former is because the feasible region of the resultant SO-DTA problem is reduced after NVH constraints are incorporated into the TSTT-TSE-SO-DTA problem. The latter is because the resultant feasible region becomes a non-convex set.

Table 5. TSTTs and TSEs obtained from different SO-DTA models in Example 3.

\begin{tabular}{rcccccc}
\hline & \multicolumn{2}{c}{ Models } & \multicolumn{2}{c}{ Without NVH constraints } & \multicolumn{2}{c}{ With NVH constraints } \\
\cline { 2 - 6 } Case & TSTT & TSE & TSTT (intervals) & TSE (g) & TSTT (intervals) & TSE (g) \\
\hline $1^{\mathrm{a}}$ & Min & - & 215 & 466.22 & 215 & 464.90 \\
$2^{\mathrm{b}}$ & - & Min & 221 & 446.90 & 221 & 446.90 \\
$3^{\mathrm{c}}$ & Min* $^{*}$ & Min & 215 & 450.86 & 215 & 463.58 \\
$4^{\mathrm{d}}$ & Min* & Max & 215 & 482.70 & 215 & 464.90 \\
$5^{\mathrm{e}}$ & Min & Min* & 218 & 446.90 & 218 & 446.90 \\
$6^{\mathrm{f}}$ & Max & Min* & 221 & 446.90 & 221 & 446.90 \\
\hline
\end{tabular}

${ }^{\mathrm{a}}$ The SO-DTA models that minimize TSTT.

${ }^{\mathrm{b}}$ The SO-DTA models that minimize TSE.

${ }^{\mathrm{c}}$ The SO-DTA models that minimize TSE subject to the minimal TSTT constraint.

${ }^{\mathrm{d}}$ The SO-DTA models that maximize TSE subject to the minimal TSTT constraint.

${ }^{\mathrm{e}}$ The SO-DTA models that minimize TSTT subject to the minimal TSE constraint.

${ }^{\mathrm{f}}$ The SO-DTA models that maximize TSTT subject to the minimal TSE constraint. 


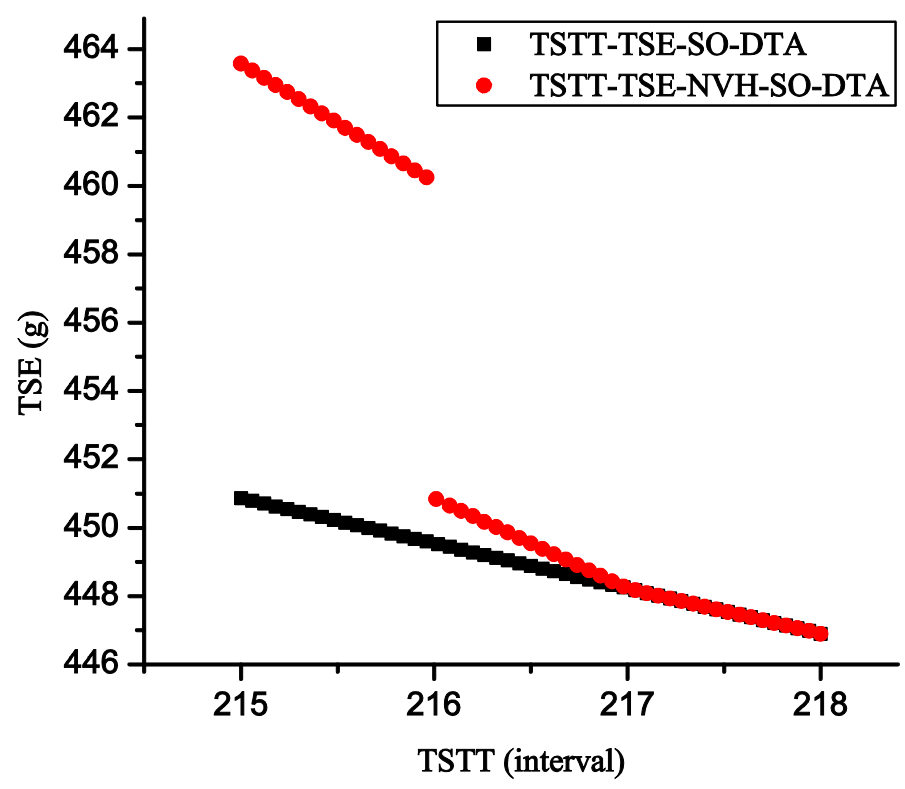

Fig. 6. The Pareto frontiers of the bi-objective SO-DTA problems in Example 3.

Example 4. SO-DTA problems in the modified Nguyen and Dupuis (1984) network: comparing the efficiency of the LTM-based models with that of the CTM-based models.

In this example, we adopt a modified Nguyen and Dupuis network (see Fig. 7) to illustrate the efficiency of the proposed LTM-based models. The network has 17 nodes, 24 links, and 2 OD pairs (form Nodes $r_{1}$ and $r_{2}$ to Node $s$ ). The length of time interval is $10 \mathrm{~s}$. The input parameter values of the LTM are the same as those in Example 1. Both OD pairs have the same OD demands, which are 15 veh/interval and last for 10 intervals. The maximum link occupancies and flow capacities of links $r_{1}-1, r_{2}-4$, and 14-s are infinite. The numbers of lanes are: 1 for Links 8-2 and 13-3, 2 for Links 12-8, 3-14, 2-14, and 9-13, and 3 for other 18 links. The length of each link is provided in Table 6, and the modeling horizon is set to be 35 intervals. 


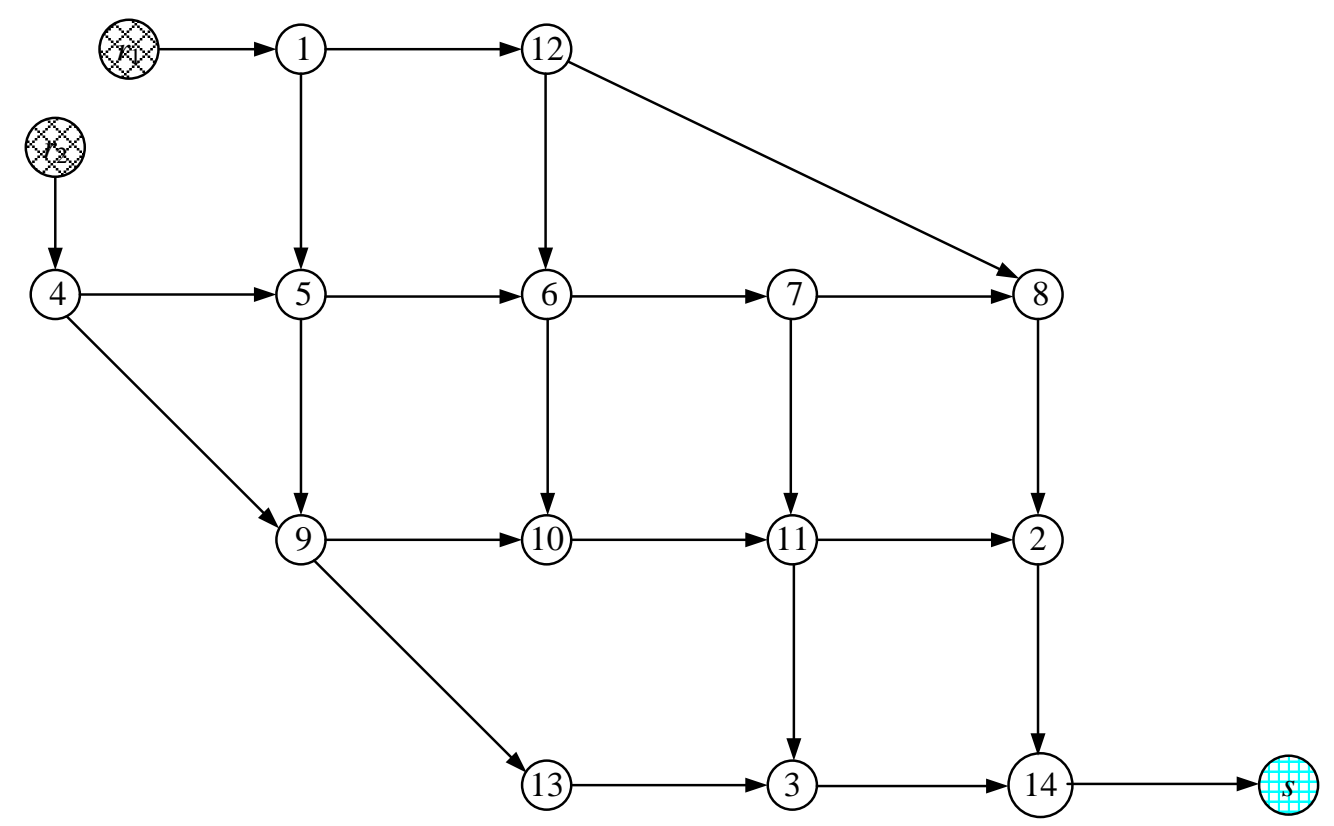

Fig. 7. The modified Nguyen and Dupuis (1984) network for Example 4.

Table 6. The length of each link in the modified Nguyen and Dupius network in Example 4.

\begin{tabular}{lccccc}
\hline Link length $(\mathrm{m})$ & 200 & 600 & 800 & 800 & 1000 \\
\hline \multirow{4}{*}{ Link } & $r_{1}-1$ & $1-12$ & $1-5$ & $4-5$ & \\
& $r_{2}-4$ & $3-14$ & $4-9$ & $5-6$ & \\
& $14-s$ & $2-14$ & $5-9$ & $6-7$ & $12-8$ \\
& & $7-8$ & $6-10$ & $9-10$ & \\
& & $7-11$ & $11-2$ & $11-3$ & \\
& $9-13$ & $12-6$ & $13-3$ & \\
& & & & \\
\hline
\end{tabular}

We both adopted the LTM and the CTM as the traffic flow models, and solved the corresponding TSTT-, TSTT-NVH-, TSE-, and TSE-NVH-SO-DTA problems. The TSTTs, the TSEs, and the CPU times required to solve different SO-DTA models are provided in Table 7. The results show that both the LTM-based and the CTM-based SO-DTA models give the same TSTT and similar TSEs. One can also observe from Table 7 that the LTM-based models have smaller problem size and obviously outperform the CTM-based models in terms of computational efficiency.

Table 7. The performance of both LTM-based and CTM-based models in Example 4.

\begin{tabular}{ccccccc}
\hline Models & & $\begin{array}{c}\text { Number of } \\
\text { Variables }\end{array}$ & $\begin{array}{c}\text { Number of } \\
\text { Constraints }\end{array}$ & $\begin{array}{c}\text { CPU Time } \\
(\mathrm{s})\end{array}$ & $\begin{array}{c}\text { TSTT } \\
(\mathrm{s})\end{array}$ & $\begin{array}{c}\text { TSE } \\
(\mathrm{g})\end{array}$ \\
\hline \multirow{2}{*}{ TSTT-SO-DTA } & LTM & 2988 & 7454 & 0.41 & 54413.33 & 10618.49 \\
& CTM & 9036 & 23497 & 1.45 & 54413.33 & 10667.61 \\
\hline
\end{tabular}




\begin{tabular}{ccccccc}
\hline \multirow{2}{*}{ TSTT-NVH-SO-DTA } & LTM & 5514 & 12215 & 1.38 & 54413.33 & 10566.61 \\
& CTM & 17442 & 38253 & 3.96 & 54413.33 & 10704.55 \\
\hline \multirow{2}{*}{ TSE-SO-DTA } & LTM & 63468 & 84974 & 6.29 & 54413.33 & 10524.21 \\
& CTM & 210636 & 329701 & 83.91 & 54500.00 & 10568.18 \\
\hline \multirow{2}{*}{ TSE-NVH-SO-DTA } & LTM & 65994 & 89735 & 30.33 & 54413.33 & 10536.06 \\
& CTM & 219042 & 344457 & 112.09 & 54510.00 & 10568.80 \\
\hline
\end{tabular}

\section{Conclusions}

In this paper, the LTM was adopted as the traffic flow model, and step functions were used to approximate link cumulative flow curves for individual links and decompose link inflow into sub-flows. We used the sub-flows to obtain the average travel time for each link inflow and sub-flow. The average link inflow travel time and the average sub-flow travel time were used to estimate average vehicle speed. We integrated average speed based emission functions to develop two methods of estimating link emissions. We proved that the link emissions obtained using the two methods converge to the same value when interval length approaches 0 . The numerical results revealed that both methods exhibit a desirable level of accuracy. Link inflow decomposition constraints were developed to retrieve sub-flows from link cumulative inflow and outflow. Considering various combinations of NVH constraints and TSTT objectives, four SO-DTA problems with environmental objectives were formulated. Examples were given to show the performance of the proposed models. The results show that the proposed SO-DTA models are more accurate than existing models, the TSTT-, TSTT-NVH-, TSE-, and TSE-NVH-SO-DTA problems can have multiple optimal solutions and the proposed the bi-objective SO-DTA models can capture the trade-off between emissions and travel time. The proposed link-based SO-DTA models also outperform cell-based models in terms of computational efficiency.

In this study, SO-DTA models were developed for single destination network applications. In future work, we will extend the proposed models to general network contexts and consider vehicle FIFO requirements. In addition, we will consider the application of the proposed SO-DTA models to the management of network wide traffic flow, such as road congestion pricing, signal control, and speed limit determination.

\section{Acknowledgements}

This work was jointly supported by the National Natural Science Foundation of China (71271075, 71431003, 71522001), the Fundamental Research Funds for the Central Universities (JZ2016HGPB0736), and a grant from the Research Grants Council of the Hong Kong Special Administrative Region, China (HKU 17201915). 


\section{Appendix A: Proof of Lemma 1}

Proof. The definition of $n_{a}^{k}$ in Eq. (8) gives the following:

$$
V_{a}\left(n_{a}^{k}-1\right) \leq U_{a}(k) \leq V_{a}\left(n_{a}^{k}\right) .
$$

Based on the relationship between the cumulative departure flow and arrival flow of link $a$, we obtain the following:

$$
\left(n_{a}^{k}-k-1\right) \delta \leq \tau_{a}(k \delta) \leq\left(n_{a}^{k}-k\right) \delta .
$$

where $\tau_{a}(k \delta)$ is the link travel time for vehicles entering link $a$ at time instant $k \delta$.

The first and second inequalities in Eq. (49), respectively, imply the following:

$$
\begin{aligned}
& \left(n_{a}^{k}-k\right) \delta \leq \tau_{a}(k \delta)+\delta, \text { and } \\
& \tau_{a}((k-1) \delta)-\delta \leq\left(n_{a}^{k-1}-k\right) \delta .
\end{aligned}
$$

Eq. (18) gives the following:

$$
t_{a}(k)=\sum_{l=1}^{\underline{K}} y_{a k}(l)(l-k) \delta / y_{a}(k)=\sum_{l=n_{a}^{k-1}}^{n_{a}^{k}} y_{a k}(l)(l-k) \delta / y_{a}(k) .
$$

Therefore,

$$
\begin{aligned}
& t_{a}(k) \geq \sum_{l=n_{a}^{k-1}}^{n_{a}^{k}} y_{a k}(l)\left(n_{a}^{k-1}-k\right) \delta / y_{a}(k)=\left(n_{a}^{k-1}-k\right) \delta, \text { and } \\
& t_{a}(k) \leq \sum_{l=n_{a}^{k-1}}^{n_{a}^{k}} y_{a k}(l)\left(n_{a}^{k}-k\right) \delta / y_{a}(k)=\left(n_{a}^{k}-k\right) \delta .
\end{aligned}
$$

Substituting Eqs. (50) and (51) into Eqs. (53) and (54), respectively, gives the following:

$$
\tau_{a}((k-1) \delta)-\delta \leq\left(n_{a}^{k-1}-k\right) \delta \leq t_{a}(k) \leq\left(n_{a}^{k}-k\right) \delta \leq \tau_{a}(k \delta)+\delta .
$$

Let $\Delta t=\delta, t=k \delta$, and $t_{a}(t, \Delta t)=t_{a}(k)$, where $t_{a}(t, \Delta t)$ is a function of $\Delta t$. Accordingly, Eq. (55) can be rewritten as follows:

$$
\tau_{a}(t-\Delta t)-\Delta t \leq t_{a}(t, \Delta t) \leq \tau_{a}(t)+\Delta t .
$$

According to Assumption 1, the continuous-time link travel time is continuous with respect to time instant $t$. Therefore,

$$
\lim _{\Delta t \rightarrow 0}\left[\tau_{a}(t-\Delta t)-\Delta t\right]=\lim _{\Delta t \rightarrow 0} \tau_{a}(t-\Delta t)=\tau_{a}(t)=\lim _{\Delta t \rightarrow 0}\left[\tau_{a}(t)+\Delta t\right] .
$$

Combining Eqs. (56) and (57) gives the following:

$$
\lim _{\Delta t \rightarrow 0} t_{a}(t, \Delta t)=\tau_{a}(t) .
$$

This implies that the link travel times calculated by Eq. (9) or (10) converge to the continuous-time 
link travel times. This completes the proof. $\square$

\section{Appendix B: Proof of Proposition 1}

Proof. The following is true by definition:

$$
\begin{aligned}
& E_{a}=\int_{0}^{T} \phi_{a}\left(L_{a} / \tau_{a}(t)\right) \tau_{a}(t) u_{a}(t) d t=\lim _{\delta \rightarrow 0} \sum_{k \in K} \int_{(k-1) \delta}^{k \delta} \phi_{a}\left(L_{a} / \tau_{a}(t)\right) \tau_{a}(t) u_{a}(t) d t \\
& =\lim _{\delta \rightarrow 0} \sum_{k \in K} \int_{(k-1) \delta}^{k \delta} \phi_{a}\left(L_{a} / \tau_{a}(k \delta)\right) \tau_{a}(k \delta) u_{a}(t) d t .
\end{aligned}
$$

According to Lemma 1, he link travel times calculated by Eq. (9) converge to the continuous-time link travel times. Therefore,

$$
\begin{aligned}
& E_{a}=\lim _{\delta \rightarrow 0} \sum_{k \in K} \phi_{a}\left(L_{a} / t_{a}(k)\right) t_{a}(k) \int_{(k-1) \delta}^{k \delta} u_{a}(t) d t \\
& =\lim _{\delta \rightarrow 0} \sum_{k \in K} \phi_{a}\left(L_{a} / t_{a}(k)\right) t_{a}(k) y_{a}(k)=\lim _{\delta \rightarrow 0} \bar{E}_{a} .
\end{aligned}
$$

This completes the proof. $\square$

\section{Appendix C: Proof of Proposition 2}

Proof. The following is true by definition:

$$
\begin{aligned}
& \lim _{\delta \rightarrow 0} \tilde{E}_{a}=\lim _{\delta \rightarrow 0} \sum_{k \in K} \sum_{l \in K} \tilde{\phi}_{a k l} y_{a}(k, l) \\
& =\lim _{\delta \rightarrow 0} \sum_{k \in K} \sum_{l \in K} \phi_{a}\left(L_{a} /(l-k) \delta\right)(l-k) \delta y_{a}(k, l) \\
& =\lim _{\delta \rightarrow 0} \sum_{k \in K} \sum_{l=n_{a}^{k-1}}^{n_{a}^{k}} \phi_{a}\left(L_{a} /(l-k) \delta\right)(l-k) \delta y_{a}(k, l) .
\end{aligned}
$$

According to Lemma 1, the link travel times calculated by Eq. (9) converge to the continuous-time link travel times. Therefore,

$$
\lim _{\delta \rightarrow 0}\left[\tau_{a}((k-1) \delta)-\delta\right]=\lim _{\delta \rightarrow 0} \tau_{a}((k-1) \delta)=\lim _{\delta \rightarrow 0} \tau_{a}(k \delta)=\lim _{\delta \rightarrow 0}\left[\tau_{a}(k \delta)+\delta\right] .
$$

Combining Inequality (55) with Eq. (62) gives the following:

$$
\lim _{\delta \rightarrow 0}(l-k) \delta=\tau_{a}(k \delta), \forall l \in\left[n_{a}^{k-1}, n_{a}^{k}\right] .
$$

Substituting Eq. (63) into Eq. (61) gives the following: 


$$
\begin{aligned}
& \lim _{\delta \rightarrow 0} \tilde{E}_{a}=\lim _{\delta \rightarrow 0} \sum_{k \in K} \sum_{l=n_{a}^{k-1}}^{n_{a}^{k}} \phi_{a}\left(L_{a} / \tau_{a}(k \delta)\right)(l-k) \delta y_{a}(k, l) \\
& =\lim _{\delta \rightarrow 0} \sum_{k \in K} \phi_{a}\left(L_{a} / \tau_{a}(k \delta)\right) \sum_{l=n_{a}^{k-1}}^{n_{a}^{k}}(l-k) \delta y_{a}(k, l) \\
& =\lim _{\delta \rightarrow 0} \sum_{k \in K} \phi_{a}\left(L_{a} / \tau_{a}(k \delta)\right) t_{a}(k) y_{a}(k) \\
& =\lim _{\delta \rightarrow 0} \sum_{k \in K} \phi_{a}\left(L_{a} / t_{a}(k)\right) t_{a}(k) y_{a}(k) \\
& =\lim _{\delta \rightarrow 0} \bar{E}_{a} .
\end{aligned}
$$

Therefore, the link emissions obtained from Eq. (16) converge to the link emissions obtained from Eq. (13). According to Proposition 1, the link emissions obtained by Eq. (13) converge to the continuous-time link emissions obtained from (2). Therefore, the link emissions obtained from Eq. (16) converge to the continuous-time link emissions obtained from (2). This completes the proof.

\section{Appendix D: Proof of Proposition 3}

Proof. We first prove that if $\mathbf{Y}$ satisfies conditions (31)-(35), then $\mathbf{Y}$ also satisfies Eq. (30). Eqs. (34) and (35) directly imply the first and third conditions in Eq. (30) are satisfied. We need only to prove that vector $\mathbf{Y}$ satisfies the second condition in Eq. (30) if $\mathbf{Y}$ satisfies conditions (31)-(35).

As $U_{a}(k)$ denotes a monotonically non-decreasing cumulative inflow, $U_{a}(k) \leq U_{a}\left(k^{\prime}-1\right)$ for all $k^{\prime} \geq k+1$ and $U_{a}(k-1) \geq U_{a}\left(k^{\prime}\right)$ for all $k^{\prime} \leq k-1$. For any interval $k$ that satisfies $U_{a}(k-1) \leq V_{a}(l) \leq U_{a}(k), \quad V_{a}(l) \leq U_{a}(k)=U_{a}((k+1)-1) \leq U_{a}\left(k^{\prime}-1\right)$ for all $k^{\prime} \geq k+1$, and $V_{a}(l) \geq U_{a}(k-1) \geq U_{a}\left(k^{\prime}\right)$ for all $k^{\prime} \leq k-1$. According to Eqs. (34) and (35), $Y_{a}\left(k^{\prime}, l\right)=0$ for all $k^{\prime} \geq k+1$ and $Y_{a}\left(k^{\prime}, l\right)=U_{a}\left(k^{\prime}\right)-U_{a}\left(k^{\prime}-1\right)$ for all $k^{\prime} \leq k-1$. Therefore, according to Eq. (33), for any intervals $k$ that satisfies $U_{a}(k-1) \leq V_{a}(l) \leq U_{a}(k)$, the following is true:

$$
\begin{aligned}
& Y_{a}(k, l)=V_{a}(l)-\sum_{k^{\prime}=1}^{k-1} Y_{a}\left(k^{\prime}, l\right)-\sum_{k^{\prime}=k+1}^{K} Y_{a}\left(k^{\prime}, l\right) \\
& =V_{a}(l)-\sum_{k^{\prime}=1}^{k-1}\left[U_{a}\left(k^{\prime}\right)-U_{a}\left(k^{\prime}-1\right)\right] \\
& =V_{a}(l)-U_{a}(k-1)+U_{a}(0) \\
& =V_{a}(l)-U_{a}(k-1) .
\end{aligned}
$$

We then prove that if $\mathbf{Y}$ satisfies Eq. (30), then $\mathbf{Y}$ also satisfies conditions (31)-(35). Eq. (30) directly implies that conditions (31), (32) (34) and (35) are satisfied. We only need to prove that condition (33) is satisfied for $\mathbf{Y}$. Because the cumulative link inflows and outflows are monotonically increasing, there are two cases should be considered: (i) an interval $k^{\prime}$ exists such that $V_{a}(l)=U_{a}\left(k^{\prime}\right)$, (ii) $V_{a}(l) \neq U_{a}(k)$ for all $k \in K$. 
In case (i), $V_{a}(l)=U_{a}\left(k^{\prime}\right) \leq U_{a}(k-1)$ for all $k>k^{\prime}$, and $V_{a}(l)=U_{a}\left(k^{\prime}\right) \geq U_{a}(k)$ for all $k \leq k^{\prime}$. According to Eq. (30), the following is true:

$$
\sum_{k \in K} Y_{a}(k, l)=\sum_{k=1}^{k^{\prime}} Y_{a}(k, l)+\sum_{k=k^{\prime}+1}^{K} Y_{a}(k, l)=\sum_{k=1}^{k^{\prime}}\left[U_{a}(k)-U_{a}(k-1)\right]=U_{a}\left(k^{\prime}\right)=V_{a}(l) .
$$

In case (ii), an interval $k^{\prime}$ exists such that $U_{a}\left(k^{\prime}-1\right)<V_{a}(l)<U_{a}\left(k^{\prime}\right)$, and $V_{a}(l)<U_{a}\left(k^{\prime}\right) \leq U_{a}(k-1)$ for all $k>k^{\prime}$ and $V_{a}(l)>U_{a}\left(k^{\prime}-1\right) \geq U_{a}(k)$ for all $k<k^{\prime}$. According to Eq. (30), the following is true:

$$
\begin{aligned}
& \sum_{k \in K} Y_{a}(k, l)=\sum_{k=1}^{k^{\prime}-1} Y_{a}(k, l)+Y_{a}\left(k^{\prime}, l\right)+\sum_{k=k^{\prime}+1}^{K} Y_{a}(k, l) \\
& =\sum_{k=1}^{k^{\prime}-1}\left[U_{a}(k)-U_{a}(k-1)\right]+V_{a}(l)-U_{a}\left(k^{\prime}-1\right) \\
& =V_{a}(l) .
\end{aligned}
$$

Condition (33) is satisfied for both cases. This completes the proof. $\square$

\section{Appendix E: Proof of Proposition 4}

Proof. We first prove that if a vector $[\mathbf{U}, \mathbf{V}, \mathbf{Y}]$ satisfies constraints (31)-(35), then a vector $\boldsymbol{\omega}$ exists such that the vector $[\mathbf{U}, \mathbf{V}, \mathbf{Y}, \boldsymbol{\omega}]$ satisfies constraint (36). Constraints (36a)-(36c) directly follows constraints (31)-(33). We set $\varpi_{a}(k, l)=0$ if $V_{a}(l)-U_{a}(k) \leq 0$, and $\varpi_{a}(k, l)=1$, otherwise. This definition of vector $\boldsymbol{\omega}$ implies that constraint (36h) is satisfied. We consider two cases: (i) $\varpi_{a}(k, l)=0$ and (ii) $\varpi_{a}(k, l)=1$. In case (i), $\varpi_{a}(k, l)=0$, $Y_{a}(k, l) \geq U_{a}(k)-U_{a}(k-1)-M=U_{a}(k)-U_{a}(k-1)-\left[1-\varpi_{a}(k, l)\right] M$ $V_{a}(l)-U_{a}(k) \leq 0=\varpi_{a}(k, l) M$, and $V_{a}(l)-U_{a}(k) \geq-M=\left[\varpi_{a}(k, l)-1\right] M$. These relationships imply that constraints (36e), (36f), and (36g) are satisfied. In case (ii), $\varpi_{a}(k, l)=1$, $V_{a}(l)-U_{a}(k)>0=\left[\varpi_{a}(k, l)-1\right] M$ and $V_{a}(l)-U_{a}(k) \leq \varpi_{a}(k, l) M$. These relationships imply that constraints (36f) and (36g) are satisfied. According to condition (35), $Y_{a}(k, l)=U_{a}(k)-U_{a}(k-1)$. This condition implies that $Y_{a}(k, l) \geq U_{a}(k)-U_{a}(k-1)$ $=U_{a}(k)-U_{a}(k-1)-\left[1-\varpi_{a}(k, l)\right] M$, and thus that constraints (36e) is satisfied. Therefore, constraints (36e), (36f), and (36g) are satisfied in both cases. Next, we consider two similar cases: (i) $\varpi_{a}(k-1, l)=0$ and (ii) $\varpi_{a}(k-1, l)=1$. In case (i), $\varpi_{a}(k-1, l)=0, V_{a}(l)-U_{a}(k-1) \leq 0$. According to condition (34), $Y_{a}(k, l)=0$. This implies that $Y_{a}(k, l) \leq 0=\varpi_{a}(k-1, l) M$, and constraint (36d) is satisfied. In case (i), $\varpi_{a}(k-1, l)=1$, constraint (36d) is also satisfied because $Y_{a}(k, l) \leq M=\varpi_{a}(k-1, l) M$. Therefore, constraint (36d) is satisfied in both cases. In sum, the vector $[\mathbf{U}, \mathbf{V}, \mathbf{Y}, \boldsymbol{\omega}]$ satisfies constraint (36). 
We then prove that the vector $[\mathbf{U}, \mathbf{V}, \mathbf{Y}]$ satisfies constraints (31)-(34) if the vector $[\mathbf{U}, \mathbf{V}, \mathbf{Y}, \boldsymbol{\omega}]$ satisfies constraint (36).We consider three cases: (i) $V_{a}(l)<U_{a}(k-1)$, (ii) $V_{a}(l)>U_{a}(k)$, and $U_{a}(k-1) \leq V_{a}(l) \leq U_{a}(k)$. In case (i), $V_{a}(l)-U_{a}(k-1)<0$. Constraints (36g) and (36h) implies that $\varpi_{a}(k-1, l)$ equals 0 . If not, $\varpi_{a}(k-1, l)=1$ and $V_{a}(l)-U_{a}(k-1) \geq\left[\varpi_{a}(k-1, l)-1\right] M=0$, which contradicts the condition that $V_{a}(l)-U_{a}(k-1)<0$. Substituting $\varpi_{a}(k-1, l)=0$ into constraint (36d) gives $Y_{a}(k, l) \geq U_{a}(k)-U_{a}(k-1)$. According to constraint (36b), $Y_{a}(k, l) \leq U_{a}(k)-U_{a}(k-1)$. Therefore, $Y_{a}(k, l)=U_{a}(k)-U_{a}(k-1)$.

Similarly, in case (ii), $V_{a}(l)-U_{a}(k)>0$. Constraints (36g) and (36h) imply that $\varpi_{a}(k, l)$ is 1 . If not, $\varpi_{a}(k, l)=0$ and $V_{a}(l)-U_{a}(k) \leq \varpi_{a}(k, l) M=0$, which contradicts the condition that $V_{a}(l)-U_{a}(k)>0$. Substituting $\varpi_{a}(k, l)=1$ into constraint (36e) gives $Y_{a}(k, l) \leq 0$. According to constraint (36a), $Y_{a}(k, l) \geq 0$.

In case (iii), $\quad U_{a}(k-1) \leq V_{a}(l) \leq U_{a}(k)$. Let $\quad k_{1}=\max \left\{k^{\prime} \mid U_{a}\left(k^{\prime}\right)<V_{a}(l)\right\} \quad$ and $k_{2}=\min \left\{k^{\prime} \mid U_{a}\left(k^{\prime}\right)>V_{a}(l)\right\}$. As $U_{a}(k)$ denotes a monotonically non-decreasing cumulative inflow, $U_{a}\left(k^{\prime}\right)<V_{a}(l)$ for all $k^{\prime} \leq k_{1}, U_{a}\left(k^{\prime}\right)=V_{a}(l)$ for all $k_{1}<k^{\prime}<k_{2}$, and $U_{a}\left(k^{\prime}\right)>V_{a}(l)$ for all $k^{\prime} \geq k_{2}$. Four more cases are considered: (iii.i) $U_{a}(k-1)=V_{a}(l)<U_{a}(k)$, (iii.ii) $U_{a}(k-1)<V_{a}(l)=U_{a}(k) \quad, \quad$ (iii.iii) $\quad U_{a}(k-1)<V_{a}(l)<U_{a}(k) \quad, \quad$ and $\quad$ (iii.iv) $U_{a}(k-1)=V_{a}(l)=U_{a}(k)$.

In case (iii.i), $k_{1}<k-1, U_{a}\left(k^{\prime}\right)=V_{a}(l)$ for all $k_{1}+1 \leq k^{\prime} \leq k-1$, and $k_{2}=k$. This implies that $U_{a}\left(k^{\prime}\right)-U_{a}\left(k^{\prime}-1\right)=0$ for all $k_{1}+2 \leq k^{\prime} \leq k-1$. According to constraint (36b), $Y_{a}\left(k^{\prime}, l\right)=0$ for all $k_{1}+2 \leq k^{\prime} \leq k-1$. According to constraint (36c), the following is true:

$$
\begin{aligned}
& Y_{a}(k, l)=V_{a}(l)-\sum_{k^{\prime}=1}^{k-1} Y_{a}\left(k^{\prime}, l\right)-\sum_{k^{\prime}=k+1}^{K} Y_{a}\left(k^{\prime}, l\right) \\
& =V_{a}(l)-\sum_{k^{\prime}=1}^{k_{1}+1} Y_{a}\left(k^{\prime}, l\right)-\sum_{k^{\prime}=k_{1}+2}^{k-1} Y_{a}\left(k^{\prime}, l\right)-\sum_{k^{\prime}=k_{2}+1}^{K} Y_{a}\left(k^{\prime}, l\right) \\
& =V_{a}(l)-\sum_{k^{\prime}=1}^{k_{1}+1} Y_{a}\left(k^{\prime}, l\right) \\
& =V_{a}(l)-\sum_{k^{\prime}=1}^{k_{1}+1}\left[U_{a}\left(k^{\prime}\right)-U_{a}\left(k^{\prime}-1\right)\right] \\
& =V_{a}(l)-U_{a}\left(k_{1}+1\right) \\
& =V_{a}(l)-U_{a}(k-1) \\
& =0 .
\end{aligned}
$$

In case (iii.ii), $k_{1}=k-1, U_{a}\left(k^{\prime}\right)=V_{a}(l)$ for all $k \leq k^{\prime} \leq k_{2}-1$, and $k_{2}=k+1$. This implies that $U_{a}\left(k^{\prime}\right)-U_{a}\left(k^{\prime}-1\right)=0$ for all $k+1 \leq k^{\prime} \leq k_{2}-1$. According to constraint (36b), $Y_{a}\left(k^{\prime}, l\right)=0$ for all $k+1 \leq k^{\prime} \leq k_{2}-1$. According to constraint (36c), the following is true: 


$$
\begin{aligned}
& Y_{a}(k, l)=V_{a}(l)-\sum_{k^{\prime}=1}^{k-1} Y_{a}\left(k^{\prime}, l\right)-\sum_{k^{\prime}=k+1}^{K} Y_{a}\left(k^{\prime}, l\right) \\
& =V_{a}(l)-\sum_{k^{\prime}=1}^{k_{1}} Y_{a}\left(k^{\prime}, l\right)-\sum_{k^{\prime}=k+1}^{k_{2}-1} Y_{a}\left(k^{\prime}, l\right)-\sum_{k^{\prime}=k_{2}}^{K} Y_{a}\left(k^{\prime}, l\right) \\
& =V_{a}(l)-\sum_{k^{\prime}=1}^{k_{1}} Y_{a}\left(k^{\prime}, l\right)-\sum_{k^{\prime}=k_{1}+2}^{k-1} Y_{a}\left(k^{\prime}, l\right)-\sum_{k^{\prime}=k_{2}+1}^{K} Y_{a}\left(k^{\prime}, l\right) \\
& =V_{a}(l)-\sum_{k^{\prime}=1}^{k_{1}}\left[U_{a}\left(k^{\prime}\right)-U_{a}\left(k^{\prime}-1\right)\right] \\
& =V_{a}(l)-U_{a}(k-1) \\
& =U_{a}(k)-U_{a}(k-1) .
\end{aligned}
$$

In case (iii.iii), $k_{1}=k-1$, and $k_{2}=k$. According to constraint (36c), the following is true:

$$
\begin{aligned}
& Y_{a}(k, l)=V_{a}(l)-\sum_{k^{\prime}=1}^{k-1} Y_{a}\left(k^{\prime}, l\right)-\sum_{k^{\prime}=k+1}^{K} Y_{a}\left(k^{\prime}, l\right) \\
& =V_{a}(l)-\sum_{k^{\prime}=1}^{k_{1}} Y_{a}\left(k^{\prime}, l\right)-\sum_{k^{\prime}=k_{2}+1}^{K} Y_{a}\left(k^{\prime}, l\right) \\
& =V_{a}(l)-\sum_{k^{\prime}=1}^{k_{1}}\left[U_{a}\left(k^{\prime}\right)-U_{a}\left(k^{\prime}-1\right)\right] \\
& =V_{a}(l)-U_{a}(k-1) .
\end{aligned}
$$

In case (iii.iv), $U_{a}(k-1)=V_{a}(l)=U_{a}(k)$, according to constraints (36a) and (36b), $Y_{a}(k, l)=0=V_{a}(l)-U_{a}(k-1)$.

In sum, the above four cases imply that $Y_{a}(k, l)=V_{a}(l)-U_{a}(k-1)$ if $U_{a}(k-1) \leq V_{a}(l) \leq U_{a}(k)$. Therefore, $Y_{a}(k, l)=U_{a}(k)-U_{a}(k-1)$ if $V_{a}(l) \leq U_{a}(k-1)$, and $Y_{a}(k, l)=0$ if $V_{a}(l) \geq U_{a}(k)$. Equivalently, $[\mathbf{U}, \mathbf{V}, \mathbf{Y}]$ satisfies constraints (34) and (35). In addition constraints (31)-(33) directly follows constraints (36a)-(36c). Therefore, [U, V, Y] satisfies constraints (31)-(35).

\section{Appendix F: Proof of Proposition 5}

Proof. According to Eqs. (6), (30) and (33), we have

$$
\begin{aligned}
& \sum_{l \in K} y_{a}(k, l)=\sum_{l \in K}\left[Y_{a}(k, l)-Y_{a}(k, l-1)\right]=Y_{a}(k, \underline{K})=U_{a}(k)-U_{a}(k-1), \text { and } \\
& \sum_{k \in K} y_{a}(k, l)=\sum_{k \in K}\left[Y_{a}(k, l)-Y_{a}(k, l-1)\right]=\sum_{k \in K} Y_{a}(k, l)-\sum_{k \in K} Y_{a}(k, l-1)=V_{a}(l)-V_{a}(l-1) .
\end{aligned}
$$

Substituting Eq. (9) into Eq. (37) gives the following:

$$
T S T T=\sum_{a \in A} \sum_{l \in K} \sum_{k \in K} y_{a}(k, l)(l-k) \delta=\delta \sum_{a \in A}\left[\sum_{l \in K} l \sum_{k \in K} y_{a}(k, l)-\sum_{k \in K} k \sum_{l \in K} y_{a}(k, l)\right] .
$$


Substituting Eqs. (65) and (66) into Eq. (67) gives the following:

$$
\begin{aligned}
& \text { TSTT }=\delta \sum_{a \in \bar{A}}\left\{\sum_{l \in K} l\left[V_{a}(l)-V_{a}(l-1)\right]-\sum_{k \in K} k\left[U_{a}(k)-U_{a}(k-1)\right]\right\} \\
& =\delta \sum_{a \in \bar{A}}\left\{\sum_{k \in K} k\left[V_{a}(k)-V_{a}(k-1)\right]-\sum_{k \in K} k\left[U_{a}(k)-U_{a}(k-1)\right]\right\} .
\end{aligned}
$$

Under Assumption 3, all vehicles arrive at destination during the studied period. This implies that all links beside destination link is empty at the end of interval $\underline{K}$, and $U_{a}(\underline{K})=V_{a}(\underline{K})$ for all $a \in \bar{A}$. Therefore,

$$
\begin{aligned}
& \sum_{k \in K} k\left[V_{a}(k)-V_{a}(k-1)\right]-\sum_{k \in K} k\left[U_{a}(k)-U_{a}(k-1)\right] \\
& =\sum_{k \in K} k\left[U_{a}(k-1)-V_{a}(k-1)\right]-\sum_{k \in K} k\left[U_{a}(k)-V_{a}(k)\right] \\
& =\sum_{k=1}^{K} k\left[U_{a}(k-1)-V_{a}(k-1)\right]-\sum_{k=1}^{K} k\left[U_{a}(k)-V_{a}(k)\right] \\
& =\sum_{k=1}^{K-1}(k+1)\left[U_{a}(k)-V_{a}(k)\right]-\sum_{k=1}^{K-1} k\left[U_{a}(k)-V_{a}(k)\right]-\underline{K}\left[U_{a}(\underline{K})-V_{a}(\underline{K})\right] \\
& =\sum_{k=1}^{K-1}\left[U_{a}(k)-V_{a}(k)\right]+\left[U_{a}(\underline{K})-V_{a}(\underline{K})\right] \\
& =\sum_{k=1}^{K}\left[U_{a}(k)-V_{a}(k)\right] \\
& =\sum_{k \in \underline{K}}\left[U_{a}(k)-V_{a}(k)\right] .
\end{aligned}
$$

Substituting Eq. (69) into Eq. (68) gives TSTT $=\delta \sum_{a \in A} \sum_{k \in \underline{K}}\left[U_{a}(k)-V_{a}(k)\right]$. This completes the proof.

\section{References}

Astarita, V., 1996. A continuous time link model for dynamic network loading models based on travel time function. In: Lesort, J.B. (Ed.). Transportation and Traffic Theory. Pergamon Elservier, New York, pp. 79-102.

Aziz, H.M., Ukkusuri, S.V., 2012. Integration of Environmental Objectives in a System Optimal Dynamic Traffic Assignment Model. Comput. Aided Civil. Infrastructure Engrg. 27 (7), 494-511.

Ban, X., Liu, H., Ferris, M.C., Ran, B., 2008. A link-node complementarity model and solution algorithm for dynamic user equilibria with exact flow propagations. Transportation Res. Part B 42 (9), 823-842.

Ban, X., Pang, J.S., Liu, X., Ma, R., 2012. Continuous-time point-queue models in dynamic network loading. Transportation Res. Part B 46 (3), 360-380.

Benedek,C.M., Rilett,L.R., 1998. Equitable traffic assignment with environmental cost functions. Journal of Transportation Engineering ASCE 124 (1), 16-22.

Carey, M., 1987. Optimal time-varying flows on congested networks. Oper. Res. 35 (1), 58-69.

Carey, M., Ge, Y.E., 2005. Convergence of a discretised travel-time model. Transportation Sci. 39 (1), 
25-38.

Carey, M., Ge, Y.E., 2012. Comparison of methods for path inflow reassignment for dynamic user equilibrium. Networks and Spatial Econom. 12 (3), 337-376.

Carey, M., Srinivasan, A. 1993. Externalities, average and marginal costs, and tolls on congested networks with time-varying flows. Oper. Res. 41 (1), 217-231.

Carey, M., Subrahmanian, E., 2000. An approach to modelling time-varying flows on congested networks. Transportation Res. Part B 34 (3), 157-183.

Carey, M., Watling, D., 2012. Dynamic traffic assignment approximating the kinematic wave model: System optimum, marginal costs, externalities and tolls. Transportation Res. Part B 46 (5), 634-648.

Chiu, Y.C., Zheng, H., Villalobos, J., Gautam, B., 2007. Modeling no-notice mass evacuation using a dynamic traffic flow optimization model. IIE Trans. 39 (1), 83-94.

Chow, A., 2007. Analysis of dynamic system optimum and externalities with departure time choice. In: Allsop, R.E., Bell, M.G.H., Heydecker, B.G. (Eds.), Proc. 17th Internat. Sympos. Transportation and Traffic Theory. New York: Elsevier, Amsterdam, pp. 301-326.

Chow, A., 2009a. Dynamic system optimal traffic assignment: A state-dependent control theoretic approach. Transportmetrica 5 (2), 85-106.

Chow, A., 2009b. Properties of system optimal traffic assignment with departure time choice and its solution method. Transportation Res. Part B 43 (3), 325-344.

Daganzo, C.F., 1995. Properties of link travel time functions under dynamic loads. Transportation Res. Part B 29 (2), 95-98.

Friesz, T.L., Bernstein, D., Smith, T.E., Tobin, R.L., Wie, B., 1993. A variational inequality formulation of the dynamic networks user equilibrium problem. Oper. Res. 41 (1), 179-191.

Friesz, T.L., Luque, J., Tobin, R.L., Wei, B.-W., 1989. Dynamic network traffic assignment considered as a continuous time optimal control problem. Oper. Res. 37 (6), 893-901.

Ghali, M.O., Smith, M.J., 1995. A model for the dynamic system optimum traffic assignment problem. Transportation Res. Part B 29 (3), 155-170.

Huang, H.J., Lam, W.H., 2002. Modeling and solving the dynamic user equilibrium route and departure time choice problem in network with queues. Transportation Res. Part B 36 (3), 253-273.

Kuwahara, M., Akamatsu, T., 2001. Dynamic user optimal assignment with physical queues for a many-to-many OD pattern. Transportation Res. Part B 35 (5), 461-479.

Lam, W.H., Huang, H.J., 1995. Dynamic user optimal traffic assignment model for many to one travel demand. Transportation Res. Part B 29 (4), 243-259.

Li, Y., Waller, S.T., Ziliaskopoulos, T., 2003. A decomposition scheme for system optimal dynamic traffic assignment models. Network Spat. Econ. 3 (4), 441-455.

Lin, W.H., Wang, C., 2004. An enhanced 0-1 mixed-integer LP formulation for traffic signal control. IEEE Trans. Intell. Transp. Syst. 5 (4), 238-245.

Liu, Y., Lai, X., Chang, G.L., 2006. Cell-based network optimization model for staged evacuation planning under emergencies. Transportation Res. Rec. 1964, 127-135.

Lo, H.K., 2001. A cell-based traffic control formulation: strategies and benefits of dynamic timing plans. Transportation Sci. 35 (2), 149-164. 
Lo, H.K., Szeto, W.Y., 2002. A cell-based variational inequality formulation of the dynamic user optimal assignment problem. Transportation Res. Part B 36 (5), 421-443.

Long, J.C., Gao, Z.Y., Szeto, W.Y., 2011. Discretised link travel time models based on cumulative flows: Formulation and properties. Transportation Res. Part B 45 (1), 232-254.

Long, J.C., Huang, H.J., Gao, Z.Y., 2013. Discretised route travel time models based on cumulative flows. J. Adv. Transportation 47(1):105-125.

Long, J.C., Szeto, W.Y., 2015. Link-based system optimum dynamic traffic assignment problems in general networks. Submitted to Oper. Res.

Ma, R., Ban, X., Szeto, W.Y., 2015. Emission modeling and pricing in dynamic traffic networks. Transportation Res. Procedia, 9, 106-129.

Ma, R., Ban, X., Pang, J.S., 2014. Continuous-time dynamic system optimum for single-destination traffic networks with queue spillbacks. Transportation Res. Part B 68, 98-122.

Meng, Q., Khoo, H.L., 2012. A computational model for the probit-based dynamic stochastic user optimal traffic assignment problem. J. Adv. Transport 46 (1), 80-94.

Merchant, D.K., Nemhauser, G.L., 1978a. A model and an algorithm for the dynamic traffic assignment. Transportation Sci. 12 (3), 183-199.

Merchant, D.K., Nemhauser, G.L., 1978b. Optimality conditions for a dynamic traffic assignment model. Transportation Sci. 12 (3), 200-207.

Munoz, J.C., Laval, J.A., 2006. System optimum dynamic traffic assignment graphical solution method for a congested freeway and one destination. Transportation Res. Part B 40 (1), 1-15.

Nagurney, A., 2000. Congested urban transportation networks and emission paradoxes. Transportation Res. Part D 5 (2), 145-151.

Newell, G.F., 1993. A simplified theory on kinematic wave in highway traffic, part I: general theory; part II: queuing at freeway bottlenecks; part III: multi-destination flows. Transportation Res. Part B 27 (4), 281-314.

Nguyen, S., Dupuis, C., 1984. An efficient method for computing traffic equilibria in networks with asymmetric transportation costs. Transportation Sci. 18 (2), 185-202.

Nie, Y., 2011. A cell-based Merchant-Nemhauser model for the system optimum dynamic traffic assignment problem. Transportation Res. Part B 45 (2), 329-342.

Pavlis, Y., Recker, W., 2009. A mathematical logic approach for the transformation of the linear conditional piecewise functions of dispersion-and-store and cell transmission traffic flow models into linear mixed-integer form. Transportation Sci. 43 (1), 98-116.

Penic, M.A., Upchurch, J., 1992. TRANSYT-7F: enhancement for fuel consumption, pollution emissions, and user costs. Transportation Res. Rec. 1360, 104-111.

Ran, B., Boyce, D., 1996. A link-based variational inequality formulation of ideal dynamic user optimal route choice problem. Transportation Res. Part C 4 (1), 1-12.

Rilett, L.R., Benedek, C.M., 1994. Traffic assignment under environmental and equity objective. Transportation Res. Rec. 1443 (1), 92-99.

Shen, W., Nie, Y., Zhang, H.M., 2007. On path marginal cost analysis and its relation to dynamic system-optimal traffic assignment. In Allsop RE, Bell MGH, Heydecker BG, ed. Proc. 17th Internat. Sympos. Transportation Traffic Theory. New York: Elsevier, pp. 327-360. 
Stein, W.R., Walker, D., 2003. Link-based calculation of motor vehicle air toxin emissions using MOBILE 6.2, Proc. Ninth TRB Conference on the Application of Transportation Plan.

Szeto, W.Y., Lo, H.K., 2004. A cell-based simultaneous route and departure time choice model with elastic demand. Transportation Res. Part B 38 (7), 593-612.

Szeto, W.Y., Lo, H.K., 2006. Dynamic traffic assignment: properties and extensions. Transportmetrica 2 (1), 31-52.

Waller, S.T., Mouskos, K.C., Kamaryiannis, D., Ziliaskopoulos, A.K., 2006. A linear model for the continuous network design problem. Comput. Aided Civil. Infrastructure Eng. 21 (5), 334-345.

Waller, S.T., Ziliaskopoulos, A.K., 2001. Stochastic dynamic network design problem. Transportation Res. Rec. 1771, 106-113.

Wardrop, J.G., 1952. Some theoretical aspects of road traffic research. Proceedings of The Institution of Civil Engineers 1 (2), 325-378.

Wie, B.W., Tobin, R.L., Carey, M., 2002. The existence, uniqueness and computation of an arc-based dynamic network user equilibrium formulation. Transportation Res. Part B 36 (10), 897-918.

Wu, J.H., Florian, M., Xu, Y.W., Rubio-Ardanaz, J.M., 1998. A projection algorithm for the dynamic network equilibrium problem. Proc. 1998 Internat. Conf. Traffic and Transportation Stud. (American Society of Civil Engineers, Reston, VA), pp. 379-390.

Yang, H., Meng, Q., 1998. Departure time, route choice and congestion toll in a queuing network with elastic demand. Transport Res. Part B 32 (4), 247-260.

Yin, Y.F., Lawphongpanich, S., 2006. Internalizing emission externality on road networks. Transportation Res. Part D 11 (4), 292-301.

Yperman, I., 2007. The link transmission model for dynamic network loading. Ph.D. dissertation, Katholieke Universiteit Leuven, Leuven, Belgium.

Zheng, H., Chiu, Y.C., 2011. A network flow algorithm for the cell-based single-destination system optimal dynamic traffic assignment problem. Transportation Sci. 45 (1), 121-137.

Zheng, H., Chiu, Y.C., Mirchandani, P.B., 2015. On the system optimum dynamic traffic assignment and earliest arrival flow problems. Transportation Sci. 49 (1), 13-27.

Zhu, F., Ukkusuri, S.V., 2013. A cell based dynamic system optimum model with non-holding back flows. Transportation Res. Part C 36, 367-380.

Ziliaskopoulos, A.K., 2000. A linear programming model for the single destination system optimum dynamic traffic assignment problem. Transportation Sci. 34 (1), 37-49. 